\title{
IMMERSIONS AND TRANSLATION STRUCTURES I: THE SPACE OF STRUCTURES ON THE POINTED DISK
}

\author{
W. PATRICK HOOPER
}

\begin{abstract}
We define a moduli space of translation structures on the open topological disk with a basepoint and endow it with a locally-compact metrizable topology. We call this the immersive topology, because it is defined using the concept of immersions: continuous maps between subsets of translation surfaces that respect the basepoints and the translation structures. Immersions induce a partial ordering on the moduli space, and we prove the ordering is nearly a complete lattice in the sense of order theory; the space is only missing a minimal element. Subsequent articles will uncover more structure and develop a topology on the space of all translation structures.
\end{abstract}

\section{INTRODUCTION}

A translation structure on a surface $\Sigma$ is an atlas of charts to the plane so that the transition functions are translations. It is a classical observation that by varying the geometry of translation structures on a surface of genus $g$ the structures can naturally converge to a translation structure on a surface of lower genus. Recent interest in studying dynamical and geometric properties of translation structures on surfaces of infinite genus has led to the need to take limits of sequences of translation surfaces whose genus is growing or limits of sequences of surfaces of infinite type whose topological type changes in the limit. In this and subsequent articles in this series, we provide a rigorous foundation for formulating such topological statements by considering the space of all translation structures (which simultaneously includes translation structures on surfaces of all topological types). This series of articles will include at least three articles:

I. In the current article, we define the immersive topology on the space of translation structures on the open disk $\Delta$ with basepoint $x_{0}$. We show this topology is locally compact and metrizable.

II. In the second article [13, we prove that the immersive topology on structures on the disk makes natural geometric and dynamical maps between translation structures on the pointed disk continuous. We also provide a practical method of proving convergence in this topology. This second article is still a work in progress, but many of the results can also be found in the earlier preprint [10].

Received by the editors May 29, 2014, and in revised form, June 2, 2015, May 27, 2016, and August 7, 2018.

2010 Mathematics Subject Classification. Primary 57M50; Secondary 30F30, 32G15, 37E99, $06 \mathrm{~B} 23$.

Support was provided by N.S.F. Grants DMS-1101233 and DMS-1500965 as well as a PSCCUNY Award (funded by The Professional Staff Congress and The City University of New York). 
III. In the third article [14, we will topologize the space of all translation structures on all surfaces with basepoint. Using the observation that the disk is homeomorphic to the universal cover of any translation surface admitting a translation structure, we push the topology on structures on the disk down to a topology on the space of all structures. This article will be an updated version of the preprint $[9]$.

\section{ANNOTATED TABLE OF CONTENTS}

We provide an annotated table of contents in order to describe the structure of this document and summarize the main results of the paper. Note that 92 and $\$ 3$ contain important definitions and concepts and should be read before setting further into the paper. You can get a sense of what is done in each section through the annotations below. Sections 4 and later are structured so that the most important results are stated in the introduction of the section, with proofs and less important results appearing later in the section.

§1: Connections And MOTIVATION ..................... page 237 We motivate this work by reviewing the classical theory of translation surfaces and discussing more recent research on translation surfaces of infinite type.

§2: THE SET OF TRANSLATION STRUCTURES ON THE DISK ........ page 239 This section describes the main objects of interest for the paper at a settheoretic level. These are the set $\tilde{\mathcal{M}}$ of isomorphism classes of translation structures on the pointed disk and the canonical (set-theoretic) disk bundle $\tilde{\mathcal{E}}$ over $\tilde{\mathcal{M}}$. The fiber in $\tilde{\mathcal{E}}$ over a point of $\tilde{\mathcal{M}}$ is naturally a pointed topological disk equipped with a translation structure in the isomorphism class of the image in $\tilde{\mathcal{M}}$. We call these fibers planar surfaces; they are equivalent to but conceptually easier to work with than the isomorphism classes of translation structures on the disk (see Proposition 4).

§3: IMMERSIONS AND A TOPOLOGY ON THE MODULI SPACE ....... page 241 An immersion between two planar surfaces is a map respecting basepoints which act as a translation in local coordinates. Whenever an immersion exists, it is unique, and we say a planar surface $P$ immerses in a planar surface $Q$. An embedding is an injective immersion. The notion of immersion and embedding yields partial orders on $\tilde{\mathcal{M}}$. We use these ideas to define the immersive topologies on $\tilde{\mathcal{M}}$ and $\tilde{\mathcal{E}}$.

§4: ThE HAUSDORFF PROPERTY $\ldots \ldots \ldots \ldots \ldots \ldots \ldots \ldots \ldots \ldots$ page 244

We develop a basic toolkit for working with immersions and embedding between planar surfaces. These tools will be used throughout the paper. We use these basic tools to prove that the immersive topologies on $\tilde{\mathcal{M}}$ and $\tilde{\mathcal{E}}$ are Hausdorff.

$\S 5:$ FUSING PLANAR SURFACES $\ldots \ldots \ldots \ldots \ldots \ldots \ldots \ldots \ldots \ldots \ldots \ldots \ldots$ page 245

The partial order defined by existence of immersions is nearly a complete lattice (in the sense of order theory): for any non-empty collection of planar surfaces, there is a minimal (in the sense of the partial order) planar surface in which every surface in the collection immerses. We call this minimal planar surface the fusion of the collection. By adding a minimal element $O$ to the space of planar surfaces, we obtain a complete lattice. That is, for any non-empty collection in $\tilde{\mathcal{M}} \cup\{O\}$ there is a maximal element of 
$\tilde{\mathcal{M}} \cup\{O\}$ which immerses in everything in the collection. We call this the core of the collection.

§: NEW OPEN SETS AND SECOND-COUNTABILITY

We introduce a tool for working with the topology. Namely, we study subsets of planar surfaces which are finite unions of rectangles. We use this to develop further intuition into the topologies. We find new natural open sets in $\tilde{\mathcal{M}}$, and prove that the immersive topologies on $\tilde{\mathcal{M}}$ and $\tilde{\mathcal{E}}$ have a countable basis.

$\S 7:$ SEQUENCES $\ldots \ldots \ldots \ldots \ldots \ldots \ldots \ldots \ldots \ldots \ldots \ldots \ldots \ldots \ldots \ldots \ldots \ldots \ldots \ldots$ page 259 Since $\tilde{\mathcal{M}}$ and $\tilde{\mathcal{E}}$ are Hausdorff and second-countable, many topological statements can be proved by considering sequences. To this end, we give necessary and sufficient conditions for sequences in these spaces to converge.

§8: ConTINUITY OF IMMERSIONS .......................... page 262

We explain that immersions and embeddings between two planar surfaces are jointly continuous in the choice of two planar surfaces constituting the domain and range of the immersion.

§9: CompaCt Subsets And METRIZABILITY ................ page 263 We prove that the only way a sequence of planar surfaces can fail to have a convergent subsequence is if the sequence of radii of the maximal Euclidean ball about the basepoints in the surfaces tends to zero. We use this fact to show that the immersive topologies on $\tilde{\mathcal{M}}$ and $\tilde{\mathcal{E}}$ are locally compact and metrizable. As an ingredient in the proof, we show sequences of planar surfaces which are increasing in the sense of immersions converge to the fusion of the sequence.

Appendix A: COMPARISON TO MCMULLEN'S GEOMETRIC TOPOLOGY ....... page 266 We contrast the immersive topology with McMullen's geometric topology.

\section{Connections And motivation}

We have taken some pains to distinguish translation structures (as defined above), from the notion of translation surface. A translation surface can be thought of as a pair $(X, \omega)$ consisting of a Riemann surface $X$ with a non-zero holomorphic 1-form $\omega$ on $X$. The 1 -form $\omega$ can be integrated to obtain charts to the plane, which are canonical up to postcomposition by translation. These charts are local homeomorphisms away from the set $Z \subset X$ of zeros of $\omega$, where cone singularities with cone angles in $2 \pi \mathbb{Z}$ appear. A translation surface $(X, \omega)$ gives rise to a translation structure on $X \backslash Z$.

Our understanding of translation surfaces has developed extensively since the pioneering work of Masur, Rauzy, Veech, and others in the late 1970s and early 1980s. The field has attracted researchers from diverse fields in mathematics including Teichmüller theory, algebraic geometry, and dynamical systems. Indeed, the interplay between these subjects has driven great progress in the field since its inception, and the field continues to be vibrant today.

We briefly discuss some well-established ideas relating to the study of the space of translation surfaces of genus $g \geq 1$, and to the convergence of a sequence of translation surfaces. For more detail see the survey articles [30, §6], 22, §2], and [34, §3.3]. Consider the moduli space of all translation surfaces of fixed genus. The collection of surfaces in this space with a fixed number of cone singularities 
with fixed cone angles is called a stratum of translation surfaces. There is a wellstudied way to place local coordinates on a stratum given via period coordinates. These coordinates give the stratum the structure of an orbifold with a locally affine structure. Of course, the cone singularities of a sequence of translation surfaces in a stratum can collide in the limit. In this case, there can still be a limiting translation surface but it lies outside the stratum. We can take such a limit by viewing the space of translation surfaces of genus $g$ as identified with the collection of pairs $(X, \omega)$, where $X$ is a Riemann surface and $\omega$ is a holomorphic 1-form. As such the space of translation surface of genus $g$ has the structure of a vector bundle over the moduli space $\mathcal{M}_{g}$ of Riemann surfaces of genus $g$. Sequences of translation surfaces of genus $g$ can still leave this space. For instance, a separating subsurface such as a cylinder could collapse to a point under a sequence. In order to take limits of such sequences, one can consider the Deligne-Mumford compactification of $\mathcal{M}_{g}$, and make appropriate considerations for corresponding degenerations of holomorphic 1-forms. See [22, Definition 4.7].

This work is primarily motivated by growing interest in the geometry and dynamics of translation surfaces of infinite topological type. Many works share a common interest in topological aspects of the space of all translation surfaces, e.g., to take limits of a sequence or to consider a continuously varying family. Examples of papers in which these ideas appear include [3, 15], and [11] for instance. This sequence of papers will provide a firm foundation for making such statements.

To provide context, it is worth noting that even elementary questions about a single translation structure on an infinite type surface can be difficult to answer. Bowman and Valdez define the singularities of a translation structure to be points in the metric completion and study their structure (and the structure of so-called "linear approaches" to the singularities) [2]. It is not yet clear how the structure of singularities relates to the topology and geometry of the surface, a topic also investigated in [28, and 4].

It is becoming increasingly clear that many of the techniques in the subject of translation surfaces are applicable to the study of infinite translation surfaces. For instance, there is widespread interest in infinite abelian branched covers of translation surfaces. Example articles include [5], [7, [17, [18, [19], 26], 27], and 29]. More relevantly, there is some work to suggest that many methods in use are applicable to infinite translation surfaces which do not arise from covering constructions. In the finite genus case, Masur's criterion [21] says that if the orbit of a translation surface under the Teichmüller flow recurs, then the vertical straight line flow is uniquely ergodic. It would be nice to have such a statement in the case where surfaces have infinite genus but finite area. There has been partial progress. The article [12] concludes ergodic theoretic results such as unique ergodicity about certain special surfaces from a notion of recurrence in the spirit of Masur's criterion. In 32 , a criterion was described for ergodicity of the straight line flow in translation surfaces of infinite topological type but finite area in terms of a rate of degeneration of a certain geometric quantity under the Teichmüller flow (showing that a topology on moduli space may not be necessary for ergodic theoretic results).

Problems in Teichmüller theory and 3-manifold topology prompted McMullen to place a geometric topology on the space of all Riemann surfaces with base frames 
paired with a quadratic differential; see [24, Appendix] and [23, §2.3]. Our approach has slightly different aims and results in a slightly different topology; see the discussion in the appendix.

Finally, the unfolding construction in polygonal billiards (due to [6] and 33]) works in the case where the polygon's angles are irrational multiples of $\pi$. We intend to use the topology to prove Conjecture 1.6 of [16. The main method of proof involves stretching these surfaces under a divergent sequence of maps in $G L(2, \mathbb{R})$ and passing to limit surfaces.

\section{The SET OF TRANSLATION STRUCTURES ON THE DISK}

2.1. Translation structures. Let $\Sigma$ denote an oriented topological surface. A translation structure on $\Sigma$ is a $(G, X)$-structure on $\Sigma$ in the sense of Thurston (see, e.g., [31]) where $G$ is the group of translations acting on $X=\mathbb{R}^{2}$, i.e., an atlas of charts $\left\{\left(U_{j}, \phi_{j}\right): j \in \mathcal{J}\right\}$ to the plane so that the transition maps are locally restrictions of translations. Here, we insist that each chart $\phi_{j}: U_{j} \rightarrow \mathbb{R}^{2}$ be orientation preserving.

Two translation structures on an oriented surface $\Sigma$ are the same if the union of the two atlases still determines a translation structure. We say the translation structures $\left\{\left(U_{j}, \phi_{j}\right): j \in \mathcal{J}\right\}$ on $\Sigma_{1}$ and $\left\{\left(V_{k}, \psi_{k}\right): k \in \mathcal{K}\right\}$ on $\Sigma_{2}$ are translation isomorphic if there is an orientation preserving homeomorphism $h: \Sigma_{1} \rightarrow \Sigma_{2}$ so that the structure determined by $\left\{\left(h\left(U_{j}\right), \phi_{j} \circ h^{-1}\right): j \in \mathcal{J}\right\}$ is the same as the structure determined by $\left\{\left(V_{k}, \psi_{k}\right): k \in \mathcal{K}\right\}$. The homeomorphism $h$ is called a translation isomorphism from the first structure to the second.

Remark 1 . We do not allow cone singularities in our translation structures. A translation structure may be obtained from a translation surface with cone singularities by removing the singular points from the underlying topological surface.

2.2. Translation structures on the pointed disk. Let $\Delta_{1}$ and $\Delta_{2}$ be oriented open topological 2-dimensional disks with basepoints $x_{1}$ and $x_{2}$, respectively. We say translation structures on these two disks are isomorphic if there is a translation isomorphism from the first structure to the second which respects basepoints. We call a translation isomorphism which respects basepoints an isomorphism.

Note that a translation structure on $\Delta_{1}$ is always isomorphic to a translation structure on $\Delta_{2}$; simply push the structure forward under an orientation preserving homeomorphism $\Delta_{1} \rightarrow \Delta_{2}$.

Remark 2 (Examples of structures on the disk). We briefly state the main construction of interest to us. If a surface $\Sigma$ admits a translation structure with a selected basepoint, then its universal cover is a topological disk with basepoint. We can then lift the translation structure to the disk. This can be done, for instance, for translation surfaces homeomorphic to a closed surface with the cone singularities removed; see Remark 1. There are other translation structures on the pointed disk as well. Any open topological disk in the plane containing the origin $\mathbf{0}$ admits a natural translation structure. This also works more generally: an open topological disk containing the basepoint in a surface with a translation structure gives a translation structure on the disk.

2.3. The set-theoretic moduli space. Throughout this paper, $\Delta$ denotes a fixed choice of an oriented open topological 2-dimensional disk with basepoint $x_{0} \in \Delta$. 
Let $\left\{\left(U_{j}, \phi_{j}\right): j \in \mathcal{J}\right\}$ be an atlas of charts determining a translation structure on $\Delta$. Because $\Delta$ is simply connected, by analytic continuation there is a unique map $\phi: \Delta \rightarrow \mathbb{R}^{2}$ so that

(1) $\phi\left(x_{0}\right)=\mathbf{0}$, where $\mathbf{0}=(0,0) \in \mathbb{R}^{2}$.

(2) For each $j \in \mathcal{J}$, the map $\left.\phi\right|_{U_{j}}$ agrees with $\phi_{j}$ up to postcomposition with a translation.

This map $\phi$ is a local homeomorphism called the developing map in the language of $(G, X)$ structures. See [31, §3.4]. Observe that the single chart $(\Delta, \phi)$ determines a translation surface structure on $\Delta$ which is the same as the original structure. Conversely, each orientation preserving local homeomorphism $\phi: \Delta \rightarrow \mathbb{R}^{2}$ determines a translation structure: the one determined by the atlas $\{(\Delta, \phi)\}$.

We will say a pointed local homeomorphism (from $\left(\Delta, x_{0}\right)$ to $\left(\mathbb{R}^{2}, \mathbf{0}\right)$ ) is an orientation preserving local homeomorphism $\phi: \Delta \rightarrow \mathbb{R}^{2}$ so that $\phi\left(x_{0}\right)=\mathbf{0}$. We use PLH to denote the collection of all such maps. Observe that our developing maps lie in PLH, and PLH is naturally identified with the collection of all translation structures on $\left(\Delta, x_{0}\right)$ modulo sameness.

Let $\mathrm{Homeo}_{+}\left(\Delta, x_{0}\right)$ denote the group of orientation preserving homeomorphisms $\Delta \rightarrow \Delta$ which fix the basepoint $x_{0}$. Note that the translation structures on $\Delta$ determined by $\phi$ and $\psi$ in PLH are isomorphic if and only if there is an $h \in$ $\mathrm{Homeo}_{+}\left(\Delta, x_{0}\right)$ so that $\psi=\phi \circ h^{-1}$. Thus, the (set-theoretic) moduli space of all translation structures on $\left(\Delta, x_{0}\right)$ modulo isomorphism is given by

$$
\tilde{\mathcal{M}}=\mathrm{PLH} / \mathrm{Homeo}_{+}\left(\Delta, x_{0}\right) .
$$

We use the notation $[\phi]$ to indicate the $\operatorname{Homeo}_{+}\left(\Delta, x_{0}\right)$-equivalence class of $\phi \in$ PLH.

Remark 3 (Quotient topology). One can endow PLH with the compact-open topology and $\tilde{\mathcal{M}}$ with the resulting quotient topology. This is not what we do in this paper, because the resulting topology is not Hausdorff. The open unit disk and the plane can be considered to be points in $\tilde{\mathcal{M}}$, and every open set containing the unit disk in the quotient topology also contains the plane.

2.4. The disk bundle over moduli space. The group $\operatorname{Homeo}_{+}\left(\Delta, x_{0}\right)$ naturally acts on PLH $\times \Delta$ by

$$
(\phi, y) \mapsto\left(\phi \circ h^{-1}, h(y)\right) \quad \text { for } h \in \operatorname{Homeo}_{+}\left(\Delta, x_{0}\right) .
$$

The canonical disk bundle over $\tilde{\mathcal{M}}$ is given by

$$
\tilde{\mathcal{E}}=(\mathrm{PLH} \times \Delta) / \mathrm{Homeo}_{+}\left(\Delta, x_{0}\right) .
$$

We denote the $\operatorname{Homeo}_{+}\left(\Delta, x_{0}\right)$-equivalence class of $(\phi, y)$ by $[\phi, y] \in \tilde{\mathcal{E}}$.

Because of the description of the $\operatorname{Homeo}_{+}\left(\Delta, x_{0}\right)$-action, there is a canonical map

$$
D e v: \tilde{\mathcal{E}} \rightarrow \mathbb{R}^{2} ; \quad[\phi, y] \mapsto \phi(y) .
$$

We call this map the (bundle-wide) developing map. There is also a natural projection from $\tilde{\mathcal{E}}$ onto the moduli space $\tilde{\mathcal{M}}$ given by

$$
\tilde{\pi}: \tilde{\mathcal{E}} \rightarrow \tilde{\mathcal{M}} ; \quad[\phi, y] \mapsto[\phi] .
$$


2.5. Structures on the fibers. We will call each fiber $\tilde{\pi}^{-1}([\phi])$ a planar surface. Observe that the choice of a representative $\phi \in[\phi]$ yields an identification of the planar surface $\tilde{\pi}^{-1}([\phi])$ with $\Delta$ :

$$
i_{\phi}: \Delta \rightarrow \tilde{\pi}^{-1}([\phi]) ; \quad y \mapsto[\phi, y] .
$$

We endow the fiber $\tilde{\pi}^{-1}([\phi])$ with the topology and orientation which make $i_{\phi}$ and orientation preserving homeomorphism and note that the selected orientation and topology is independent of the choice of $\phi \in[\phi]$. The map $i_{\phi}$ can also be used to push the basepoint $x_{0} \in \Delta$ onto the planar surface. We treat $\left[\phi, x_{0}\right]$ as the basepoint of the planar surface, and note that this point is also independent of the choice of $\phi$. Finally, we note that the restriction of the developing map to the fiber is an orientation preserving local homeomorphism to the plane which sends the basepoint $\left[\phi, x_{0}\right]$ to $\mathbf{0}$. This endows the fiber $\tilde{\pi}^{-1}([\phi])$ with the structure of a translation surface (using an atlas consisting of only the developing map restricted to the fiber). The translation structure on the fiber $\tilde{\pi}^{-1}([\phi])$ with basepoint $\left[\phi, x_{0}\right]$ is isomorphic to those in the pointed translation structures in the equivalence class $[\phi]$.

These ideas lead to the following result.

Proposition 4. Any translation structure on an oriented open 2-dimensional topological disk with basepoint is isomorphic to a unique planar surface.

Notational Convention 5. We follow some conventions to simplify notation by effectively removing the need to discuss equivalence classes. Formally, the planar surfaces are parametrized by equivalence classes $[\phi] \in \tilde{\mathcal{M}}$ via $P=\tilde{\pi}^{-1}([\phi])$. We will identify the objects $P$ and $[\phi]$, and thus we can more simply write $P \in \tilde{\mathcal{M}}$. We will typically denote the basepoint of $P$ by $o_{P}$; as noted above $o_{P}=\left[\phi, x_{0}\right]$. We will denote points of $P$ by letters such as $p, q \in P$. Note that points of $P$ are also points of $\tilde{\mathcal{E}}$. But, we will rarely refer to a point $p \in \tilde{\mathcal{E}}$ without referring to the planar surface $P=\tilde{\pi}(p) \in \tilde{\mathcal{M}}$ which contains $p$. Therefore, we will redundantly refer to points of $\tilde{\mathcal{E}}$ as pairs $(P, p)$ where $P$ is a planar surface and $p \in P$ is a point in this surface (i.e., $P=\tilde{\pi}(p)$ ).

\section{IMMERSiONS AND A TOPOLOGY ON THE MODULI SPACE}

3.1. Definition of immersion. Let $P$ be a planar surface, following the discussion in 92 including Convention 5 . We define $\mathrm{PC}(P)$ to be the collection of all pathconnected subsets of $P$ which contain the basepoint $o_{P} \in P$.

Let $P$ and $Q$ be planar surfaces, and choose $A \in \mathrm{PC}(P)$ and $B \in \mathrm{PC}(Q)$. We say " $A$ immerses into $B$ " and write " $A \rightsquigarrow B$ " if there is a continuous map $\iota: A \rightarrow B$ which acts as a translation in local coordinates so that $\iota\left(o_{P}\right)=o_{Q}$. We call the map $\iota$ an immersion (respecting the translation structures). We will write " $A \not \rightarrow B$ " to indicate that $A$ does not immerse in $B$, and will write " $\exists \iota: A \rightsquigarrow B$ " as shorthand for the phrase "there exists an immersion $\iota$ from $A$ to $B$ ".

Example 6. If one takes a finite genus translation surface with singularities removed, the developing map applied to the universal cover is an immersion of this cover into the plane.

An embedding is an injective immersion. If such a map exists between an $A \in$ $\mathrm{PC}(P)$ and $B \in \mathrm{PC}(Q)$, we say " $A$ embeds in $B$ " and write " $A \hookrightarrow B$ ". We follow notational conventions as for immersions. 
Next we make some basic observations about immersions.

Proposition 7 (Uniqueness of immersions). For $A \in P C(P)$ and $B \in P C(Q)$, there is at most one immersion from $A$ into $B$.

Proof. Suppose $\iota_{1}$ and $\iota_{2}$ are immersions from $A$ to $B$. We will show that $\iota_{1}(a)=$ $\iota_{2}(a)$ for all $a \in A$. The set of $a$ satisfying this equation is open (because both $\iota_{1}$ and $\iota_{2}$ are local translations) and closed (because of continuity of the maps). Also it holds at $o_{P} \in A$ by definition of immersion. Since $A$ is path connected, it must hold in all of $A$.

An isomorphism between $A \in \mathrm{PC}(P)$ and $B \in \mathrm{PC}(Q)$ is an immersion of $A$ into $B$ which is also a homeomorphism. Compositions and inverses of isomorphisms are still isomorphisms. This implies that the notion of isomorphism gives an equivalence relation on $\bigcup_{P \in \tilde{\mathcal{M}}} \mathrm{PC}(P)$ and we use $\mathrm{PC}$ to denote the collection of such isomorphism classes. Observe that if $A$ and $A^{\prime}$ are isomorphic and $B$ and $B^{\prime}$ are isomorphic, then $A \rightsquigarrow B$ if and only if $A^{\prime} \rightsquigarrow B^{\prime}$. Therefore, the notion of immerses gives a relation on the collection $\mathrm{PC}$ of isomorphism classes of elements of $\bigcup_{P \in \tilde{\mathcal{M}}} \mathrm{PC}(P)$.

Remark 8 . We will abuse notation by typically ignoring the distinction between an $A \in \mathrm{PC}(P)$ and its equivalence class in PC. If $A \in \mathrm{PC}$, then a point in $A$ would formally be an isomorphism class of pairs $(A, a)$ much as in our definition of $\tilde{\mathcal{E}}$. We are not interested in giving more structure to $\mathrm{PC}$, so we will ignore this issue.

Corollary 9 (Partial ordering). The notions of immerses $(\rightsquigarrow)$ and embeds $(\hookrightarrow)$ viewed as relations on $P C$ are partial orderings. Both notions restrict to partial orderings on $\tilde{\mathcal{M}}$.

Proof. Let $A, B, C \in \mathrm{PC}$. The identity map is an embedding $A \hookrightarrow A$, so $\rightsquigarrow$ and $\hookrightarrow$ are reflective. If $A \rightsquigarrow B$ and $B \rightsquigarrow C$, then the composition of immersions gives an immersion $A \rightsquigarrow C$, so $\rightsquigarrow$ is transitive. Furthermore, if both maps are injective, then so is the composition, so $\hookrightarrow$ is transitive. We must show that $A \rightsquigarrow B$ and $B \rightsquigarrow A$ implies $A$ and $B$ are isomorphic. Let $\iota_{1}: A \rightsquigarrow B$ and $\iota_{2}: B \rightsquigarrow A$. Then $\iota_{2} \circ \iota_{1}$ is an immersion of $A$ into itself. Since the identity map on $A$ is also an immersion of $A$ into itself, by the uniqueness of immersions, $\iota_{2} \circ \iota_{1}=i d_{A}$. Similarly, $\iota_{1} \circ \iota_{2}=$ $i d_{B}$. The immersions are inverses of one another, and hence are homeomorphisms. Furthermore, the fact that the maps respect the basepoints implies that they are isomorphisms. This also holds for embeddings since embeddings are a special case of immersions.

Proposition 10. Let $P$ and $Q$ be planar surfaces and let $A \in P C(P)$ and $B \in$ $P C(P)$. A continuous function $f: A \rightarrow B$ satisfying $f\left(o_{P}\right)=o_{Q}$ is an immersion if and only if $\operatorname{Dev}(P, a)=\operatorname{Dev}(Q, f(a))$ for all $a \in A$.

As a consequence of this, we see that the developing map is well-defined when restricted to an $A \in \mathrm{PC}$. That is, if $A \in \mathrm{PC}(P)$ and $B \in \mathrm{PC}(Q)$ are isomorphic, then the isomorphism does not affect the image under the developing map.

Proof. Clearly if $\operatorname{Dev}(P, a)=\operatorname{Dev}(Q, f(a))$ holds for all $a \in A$, then $f$ is a local translation and so is an immersion. Conversely suppose $f$ is an immersion. Note that the developing map of a planar surface $\left.D e v\right|_{P}: P \rightarrow \mathbb{R}^{2}$ is an immersion if $\mathbb{R}^{2}$ is considered to be a planar surface with basepoint $\mathbf{0}$. Since compositions 
of immersions are immersions, we know Dev $\circ f$ is an immersion of $A$ into $\mathbb{R}^{2}$. Therefore, this map agrees with the restriction of Dev to $A$.

3.2. The topology on moduli space. We will specify the topology on the moduli space of all planar surfaces, $\tilde{\mathcal{M}}$, by specifying a subbasis for the topology. That is, we will be concerned with the coarsest topology which makes a collection of sets open.

Let $P \in \tilde{\mathcal{M}}$ be a planar surface and let $K \in \mathrm{PC}(P)$ be a compact subset of $P$. We define the following subsets of the moduli space $\tilde{\mathcal{M}}$ :

$$
\tilde{\mathcal{M}}_{\rightsquigarrow}(K)=\{Q \in \tilde{\mathcal{M}}: K \rightsquigarrow Q\} \quad \text { and } \quad \tilde{\mathcal{M}}_{\hookrightarrow}(K)=\{Q \in \tilde{\mathcal{M}}: K \hookrightarrow Q\} .
$$

Sets of this form will be open in our topology. However, they are insufficient to form a subbasis for a Hausdorff topology, because they fail to isolate points. For instance, the plane (interpreted as a planar surface) lies in each set $\tilde{\mathcal{M}}_{\rightsquigarrow}(K)$. Also, any set of the form $\tilde{\mathcal{M}}_{\hookrightarrow}(K)$ which contains the unit disk also contains the plane.

Let $P$ be a planar surface, and let $U \in \mathrm{PC}(P)$ be open as a subset of $P$. We define:

$$
\tilde{\mathcal{M}}_{\nsim}(U)=\{Q \in \tilde{\mathcal{M}}: U \not \leftrightarrow Q\} \quad \text { and } \quad \tilde{\mathcal{M}}_{\hookrightarrow}(U)=\{Q \in \tilde{\mathcal{M}}: U \nrightarrow Q\} .
$$

These sets will also be open in our topology.

We would like to describe a subbasis for our topology which consists of sets which are fairly easy to work with. So, for any planar surface $P$, we will distinguish two natural subsets of $\mathrm{PC}(P)$. We define $\overline{\operatorname{Disk}}(P)$ to be those sets in $\mathrm{PC}(P)$ which are homeomorphic to a closed 2-dimensional disk and contain the basepoint $o_{P}$ in their interior. We define $\operatorname{Disk}(P)$ to be the set of sets in $\mathrm{PC}(P)$ which are homeomorphic to an open 2-dimensional disk and contain the basepoint.

Definition 11. The immersive topology on $\tilde{\mathcal{M}}$ is the coarsest topology so that sets of either of the two forms below are open:

(1) sets of the form $\tilde{\mathcal{M}}_{\rightsquigarrow}(K)$, where $K \in \overline{\operatorname{Disk}}(P)$ for some $P \in \tilde{\mathcal{M}}$;

(2) sets of the form $\tilde{\mathcal{M}}_{\hookrightarrow}(U)$, where $U \in \operatorname{Disk}(P)$ for some $P \in \tilde{\mathcal{M}}$.

It will follow that the other types of sets mentioned above are also open; see 6 ,

3.3. The topology on the canonical disk bundle. We recall that in Convention [5] we have identified the canonical disk bundle $\tilde{\mathcal{E}}$ with the collection of pairs $(P, p)$, where $P$ is a planar surface and $p \in P$.

Let $P$ be a planar surface, and let $K \in \mathrm{PC}(P)$ be a compact subset of $P$ with interior $K^{\circ}$. Let $U \subset K^{\circ}$ be an open set (not necessarily connected or containing the basepoint). Define the following subset of $\tilde{\mathcal{E}}$ :

$$
\tilde{\mathcal{E}}_{\rightsquigarrow}(K, U)=\{(Q, q) \in \tilde{\mathcal{E}}: \exists \iota: K \rightsquigarrow Q \text { and } q \in \iota(U)\} .
$$

The immersive topology on $\tilde{\mathcal{E}}$ is the coarsest topology so that the projection $\tilde{\pi}$ : $\tilde{\mathcal{E}} \rightarrow \tilde{\mathcal{M}}$ is continuous and so that for each $P \in \tilde{\mathcal{M}}$ and each $K \in \overline{\operatorname{Disk}}(P)$ and each open $U \subset K^{\circ}$, the set $\tilde{\mathcal{E}}_{\rightsquigarrow}(K, U)$ is open.

The following results follow quickly from the following definition.

Proposition 12. Let $P$ be a planar surface. Then $P$ inherits a topology by viewing $P$ as a subset of $\tilde{\mathcal{E}}$ with the immersive topology. This topology is the same as the topology coming from viewing $P$ as homeomorphic to a topological disk as in $\$ 2.5$. 
Proof. Let $\mathcal{T}$ be the first topology on $P$ mentioned. Then open sets in $\mathcal{T}$ are of the form $\iota(U)$ where $\iota: K \rightarrow P$ is an immersion of a compact set. Since immersions are local homeomorphisms, $\iota(U)$ is always open in the disk topology. On the other hand if $V$ is an open set in the disk topology on $P$ and $v \in V$, then we can find a compact set $K \subset P$ containing $v$ in its interior, and we we can let $U=K^{\circ} \cap V$. Then the intersection of $\tilde{\mathcal{E}}_{\rightsquigarrow}(K, U)$ with $P$ is precisely $U$. Since this can be done for any $v \in V$, this shows $V$ is open in $\mathcal{T}$.

Sets $\tilde{\mathcal{E}}_{\rightsquigarrow}(K, U)$ are open even if the condition that $K$ be a closed disk is removed.

Proposition 13. For every compact $K$ in some $P$, and every $U \subset K^{\circ}$, we have $\tilde{\mathcal{E}}_{\rightsquigarrow}(K, U)$ is open.

Proof. Let $(Q, q) \in \tilde{\mathcal{E}}_{\rightsquigarrow}(K, U)$. Then there is an immersion $\iota: K \rightsquigarrow Q$ and $q \in \iota(U)$. Choose $D \in \overline{\operatorname{Disk}}(Q)$ so that $\iota(K) \subset D^{\circ}$. Then $\tilde{\mathcal{E}}_{\rightsquigarrow}(D, \iota(U))$ is open by definition and contains $(Q, q)$. We claim that $\tilde{\mathcal{E}}_{\rightsquigarrow}(D, \iota(U)) \subset \tilde{\mathcal{E}}_{\rightsquigarrow}(K, U)$ which will prove that $\tilde{\mathcal{E}}_{\rightsquigarrow}(K, U)$ is open. Let $(R, r) \in \tilde{\mathcal{E}}_{\rightsquigarrow}(D, \iota(U))$. Then there is an immersion $j: D \rightsquigarrow R$ and $r \in j \circ \iota(U)$. By composition we have an immersion $j \circ \iota: K \rightsquigarrow R$ and since $r \in j \circ \iota(U)$ we know $(R, r) \in \tilde{\mathcal{E}}_{\rightsquigarrow}(K, U)$.

\section{The HausdorfF PROPERTY}

We prove the following lemma.

Lemma 14. The immersive topologies on $\tilde{\mathcal{M}}$ and $\tilde{\mathcal{E}}$ are Hausdorff topologies.

4.1. Basic properties of immersions. We collect some basic properties of immersions and embeddings.

Proposition 15. Let $P$ and $Q$ be planar surfaces and $B \in P C(Q)$. Suppose that $\left\langle A_{j} \in P C(P)\right\rangle_{j \in \mathbb{N}}$ is an increasing sequence of open subsets, i.e., $A_{j} \subset A_{j+1}$ for all $j \in \mathbb{N}$. Let $U=\bigcup_{j \in \mathbb{N}} A_{j} \in P C(P)$. Then:

- If $A_{j} \rightsquigarrow B$ for all $j \in \mathbb{N}$, then $U \rightsquigarrow B$.

- If $A_{j} \hookrightarrow B$ for all $j \in \mathbb{N}$, then $U \hookrightarrow B$.

Proof. Suppose $\exists \iota_{j}: A_{j} \rightsquigarrow B$ for all $j \in \mathbb{N}$. The fact that these immersions are unique implies that for all $j<k$ and all $p \in A_{j}$, we have $\iota_{j}(p)=\iota_{k}(p)$, since $\iota_{j}=\left.\iota_{k}\right|_{A_{j}}$. Therefore, we may define a limiting map $\iota: U \rightarrow B$ by $\iota(p)=\iota_{j}(p)$ whenever $p \in A_{j}$. The preceding argument indicates that this map is well-defined. We must check that it is an immersion. Because the $A_{j}$ are open, continuity of each $\iota_{j}$ implies continuity of $\iota$. Similarly, $\iota$ acts as a translation in local coordinates by restricting locally within some $A_{j}$. The embedding case also requires checking injectivity. To prove this from the fact that each $\iota_{j}$ is injective, choose any distinct $p, q \in U$. Then $p, q \in \iota_{j}\left(A_{j}\right)$ for some $j$. Then by definition of $\iota$ and injectivity of $\iota_{j}$,

$$
\iota(p)=\iota_{j}(p) \neq \iota_{j}(q)=\iota(q) .
$$

4.2. Subsets homeomorphic to disks. In order to work with disks in planar surfaces, we will utilize some structure coming from Schoenflies' theorem.

Theorem 16 (Schoenflies). Let $C$ be a simple closed curve in the open topological disk $\Delta$. Then, there is a homeomorphism $h: \Delta \rightarrow \mathbb{R}^{2}$ so that $h(C)$ is the unit circle in $\mathbb{R}^{2}$. 
We translate this theorem into our setting as follows.

Corollary 17. Let $P$ be a planar surface and $K \in \overline{\operatorname{Disk}}(P)$. Then, there is a homeomorphism $h: P \rightarrow \mathbb{R}^{2}$ so that $h(K)$ is the closed unit ball and $h\left(o_{P}\right)=\mathbf{0}$.

We use this to impart the following structure.

Proposition 18. Let $P$ be a planar surface. For each set $K \in \overline{D i s k}(P)$, there is a family of sets $\left\{K_{t} \in \overline{\operatorname{Disk}}(P): t>0\right\}$ so that the following statements hold:

(1) $K_{1}=K$.

(2) $\bigcap_{t} K_{t}=\left\{o_{P}\right\}$.

(3) $P=\bigcup_{t} K_{t}^{\circ}$.

(4) For each $t>0, K_{t}^{\circ}=\bigcup_{t^{\prime}<t} K_{t^{\prime}}^{\circ}$.

(5) For each $t>0, K_{t}=\bigcap_{t^{\prime}>t} K_{t^{\prime}}$.

(6) There is a continuous surjective function $\alpha: \mathbb{R} / 2 \pi \mathbb{Z} \times[0, \infty) \rightarrow P$, which is injective except that $\alpha(\mathbb{R} / 2 \pi \mathbb{Z} \times\{0\})=\left\{o_{P}\right\}$ and satisfies $\alpha(\mathbb{R} / 2 \pi \mathbb{Z} \times\{t\})=$ $\partial K_{t}$.

Proof. Let $h: P \rightarrow \mathbb{R}^{2}$ be the homeomorphism guaranteed to exist by Corollary 17. The family given by $K_{t}=h^{-1}\left(\left\{\mathbf{v} \in \mathbb{R}^{2}:\|\mathbf{v}\| \leq t\right\}\right)$ satisfies the proposition. The function $\alpha$ can be taken to be the pull back of polar coordinates on $\mathbb{R}^{2}$.

We will call any family of sets $\left\{K_{t} \in \overline{\operatorname{Disk}}(P): t>0\right\}$ formed as above a closed disk family in the planar surface $P$. Closed disk families give a natural way to understand immersions and the failure to immerse, and we use them throughout the paper. The next subsection uses them to prove our convergence criteria.

\subsection{Proofs of the Hausdorff property.}

Proof that the immersive topology on $\tilde{\mathcal{M}}$ is Hausdorff. Let $P, Q \in \tilde{\mathcal{M}}$ be distinct planar surfaces. Then by Corollary [9, either $P \not \hookrightarrow Q$ or $Q \not \rightarrow P$. Without loss of generality, assume $P \not \sim Q$. Let $\left\{K_{t}\right\}$ be a closed disk family for $P$. If each $K_{t}^{\circ} \rightsquigarrow Q$, then $P \rightsquigarrow Q$ by Proposition 15. Therefore, there is a $t$ so that $K_{t}^{\circ} \leftrightarrow Q$. We conclude that $P \in \tilde{\mathcal{M}}_{\rightsquigarrow}\left(K_{t}\right)$ and $Q \in \tilde{\mathcal{M}}_{\varkappa}\left(K_{t}^{\circ}\right)$. These open sets are disjoint since if $K_{t} \rightsquigarrow R$, then restriction gives an immersion $K_{t}^{\circ} \rightsquigarrow R$.

Proof that the immersive topology on $\tilde{\mathcal{E}}$ is Hausdorff. Suppose $(P, p)$ and $(Q, q)$ are distinct points in $\tilde{\mathcal{E}}$. We will find open sets separating these points. If $P$ and $Q$ are distinct planar surfaces, then we can use the Hausdorff property of the embedding topology on $\tilde{\mathcal{M}}$ to separate $P$ and $Q$ by open sets. The preimages of these open sets under $\tilde{\pi}$ are open in $\tilde{\mathcal{E}}$ and separate our points.

Otherwise, we have $P=Q$, and $q \in P$. In this case, the points can be separated since the induced topology on the planar surface $P$ is the same as the topology of an open topological disk; see Proposition 12.

\section{FUSING PLANAR SURFACES}

By Corollary 9 the notion of immersion places a partial ordering on the space $\tilde{\mathcal{M}}$ of planar surfaces. We will now describe some of the order structure. If $P \rightsquigarrow Q$ we think of $Q$ as larger than $P$. The content of the following is that every collection of planar surfaces has a least upper bound. 

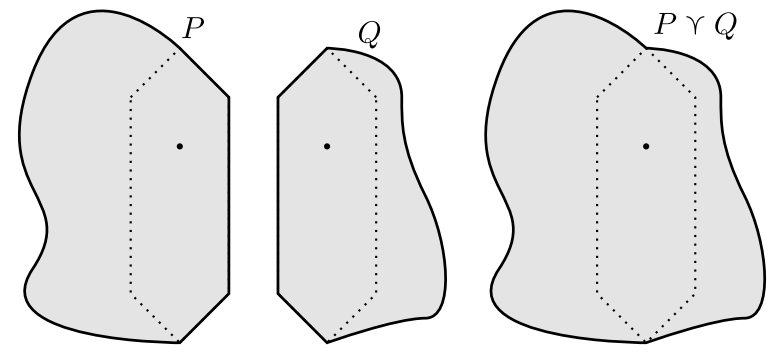

FiguRE 1. An example of fusing two planar surfaces.

Theorem 19 (Fusion Theorem). Let $\mathcal{P}$ denote any non-empty collection of planar surfaces. Then there is a unique planar surface $R$ which satisfies the following statements:

(I) For each $P \in \mathcal{P}, P \rightsquigarrow R$.

(II) For all planar surfaces $Q$, if $P \rightsquigarrow Q$ for all $P \in \mathcal{P}$, then $R \rightsquigarrow Q$.

We say that the planar surface $R$ from the above theorem is the fusion of $\mathcal{P}$ and write $R=\curlyvee \mathcal{P}$. If $\mathcal{P}$ is a finite collection, such as $\mathcal{P}=\left\{P_{1}, P_{2}, P_{3}\right\}$, we write $P_{1} \curlyvee P_{2} \curlyvee P_{3}$ for the fusion. See Figure 1 for an example of this operation.

We will now look at greatest lower bounds. Unfortunately, there is no minimal element in $\tilde{\mathcal{M}}$. We can fix this by adding a new element; define $\overline{\mathcal{M}}=\tilde{\mathcal{M}} \cup\{O\}$ and extend the partial order $\rightsquigarrow$ by saying $O \rightsquigarrow P$ for all $P \in \overline{\mathcal{M}}$ and $P \rightsquigarrow O$ if and only if $P=O$. Now $O$ is the minimal element of $\overline{\mathcal{M}}$. All subcollections of $\overline{\mathcal{M}}$ have greatest lower bounds.

Corollary 20 (Core corollary). Let $\mathcal{P} \subset \overline{\mathcal{M}}$ be non-empty. Then, there is a unique $R \in \overline{\mathcal{M}}$ which satisfies the following statements:

(I') For each $P \in \mathcal{P}, R \rightsquigarrow P$.

(II') If $Q \in \overline{\mathcal{M}}$ and $Q \rightsquigarrow P$ for all $P \in \mathcal{P}$, then $Q \rightsquigarrow R$.

The proof is a standard observation in order theory, but we give it for completeness. This and the result above indicate that $\overline{\mathcal{M}}$ is a complete lattice in the sense of order theory. See [1] or [8] for background on complete lattices.

Proof. First we address uniqueness. Suppose $R_{1}$ and $R_{2}$ satisfy both statements. Then by (I'), $R_{1} \rightsquigarrow P$ and $R_{2} \rightsquigarrow P$ for all $P \in \mathcal{P}$. Then by (II'), $R_{1} \rightsquigarrow R_{2}$ and $R_{2} \rightsquigarrow R_{1}$. We conclude that $R_{1}=R_{2}$ since $\rightsquigarrow$ is a partial order.

Consider the collection $\mathcal{S}=\{S \in \tilde{\mathcal{M}}: S \rightsquigarrow P$ for all $P \in \mathcal{P}\}$. If this collection is empty, then we can take $R=O$. The statements (I') and (II') are clearly satisfied.

If $\mathcal{S} \neq \emptyset$, then let $R=\curlyvee \mathcal{S}$. We will now prove that (I') is satisfied. Fix $P \in \mathcal{P}$. Observe that $S \rightsquigarrow P$ for all $S \in \mathcal{S}$. Therefore $R \rightsquigarrow P$ by statement (II) of the Fusion Theorem. We now prove that (II') is satisfied. Suppose $Q \in \overline{\mathcal{M}}$ and suppose $Q \rightsquigarrow P$ for every $P \in \mathcal{P}$. Then $Q \in \mathcal{S}$. So $Q \rightsquigarrow R$ by statement (I) of the Fusion Theorem.

We call the $R \in \overline{\mathcal{M}}$ produced in the above corollary the core of the collection $\mathcal{P}$, and denote this by $R=\curlywedge \mathcal{P}$. 

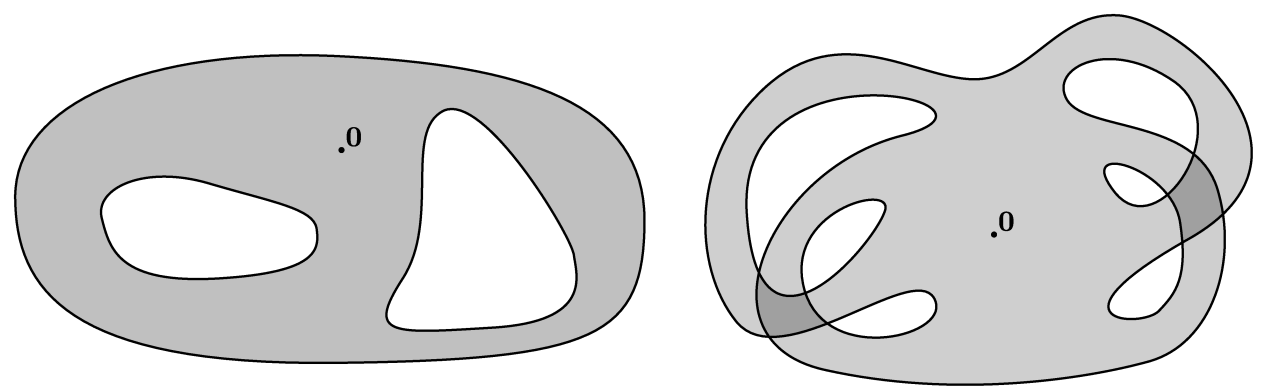

FiguRE 2. Trivial structures on the thrice punctured sphere and once punctured genus two surface.

5.1. Trivial structures. Let $\Sigma$ be a connected oriented topological surface with basepoint $x_{0}$. We will say that a trivial structure on a surface is an atlas of orientation preserving local homeomorphisms (charts) to the plane so that the transition functions are restrictions of the identity map on the plane and so that the image of the basepoint $x_{0}$ is always mapped to $\mathbf{0} \in \mathbb{R}^{2}$. We emphasize that in a trivial structure, the underlying topological surface $\Sigma$ need not be a topological disk.

We give some examples of trivial surfaces to orient the reader. Any connected open subset of $\mathbb{R}^{2}$ containing the origin $\mathbf{0}$ is a trivial surface, where it suffices to take the identity map as the sole chart in the atlas. (See Figure 2, ) Considering the developing map as the sole chart for a planar surface yields a trivial structure on any planar surface. A trivial structure is a special type of translation structure, i.e., for every translation structure there is at most one corresponding trivial structure. Furthermore, a translation structure on a surface $S$ admits a compatible trivial structure if and only if the holonomy map $\pi_{1}(S) \rightarrow \mathbb{R}^{2}$ has trivial image. So, in particular, given any translation structure on a surface, there is a compatible trivial structure on its universal abelian cover.

We will see that trivial structures generalize but share a lot of properties of translation structures on disks. Fix a translation structure on a pointed topological surface $\Sigma$. Because the transition functions are the identity map, the image of a point $y \in \Sigma$ under a chart is independent of the choice of the chart. It follows that a trivial structure on a surface can be specified by a single chart, a single orientation preserving local homeomorphism $\phi: \Sigma \rightarrow \mathbb{R}^{2}$ so that $\phi\left(x_{0}\right)=\mathbf{0}$ (which generalizes the developing map for a translation structure on a disk). Two such local homeomorphisms $\phi$ and $\psi$ are said to yield isomorphic trivial structures if there is an orientation preserving homeomorphism $h: \Sigma \rightarrow \Sigma$ so that $h\left(x_{0}\right)=x_{0}$ and $\phi \circ$ $h^{-1}=\psi$. The homeomorphism $h$ is called an isomorphism between the structures. The (set-theoretic) moduli space of trivial structures on $\left(\Sigma, x_{0}\right)$ is the collection isomorphism-equivalence classes of trivial structures on $\left(\Sigma, x_{0}\right)$. We can construct a canonical $\Sigma$-bundle over the moduli space of trivial structures on $\left(\Sigma, x_{0}\right)$, as for translation structures on the disk. We say that a trivial surface (homeomorphic to $\left.\left(\Sigma, x_{0}\right)\right)$ is a fiber of the projection from the bundle to the moduli space. We endow the trivial surfaces with a pointed trivial structure in the canonical way. This choice makes the trivial structure on the fiber an element of the isomorphism class described by the image in moduli space. 
The collection of all trivial surfaces is the collection of fibers of such projections taken over all homeomorphism-equivalence classes of pointed surfaces $\left(\Sigma, x_{0}\right)$. The developing map Dev is well-defined on the union of all trivial surfaces, and the restriction to a single trivial surface is a local homeomorphism to $\mathbb{R}^{2}$ which carries the basepoint to $\mathbf{0}$.

The notions of immersion and embedding carry over trivially to subsets of trivial surfaces. In particular, we note that if $P$ and $Q$ are trivial surfaces, then $P \rightsquigarrow Q$ and $Q \rightsquigarrow P$ implies that $P=Q$.

5.2. The Fusion Theorem. The main goal of this section is to prove the following theorem.

Theorem 21 (Generalized Fusion Theorem). Let $\mathcal{P}$ denote any non-empty collection of trivial surfaces. Then there is a unique trivial surface $R$ which satisfies the following statements:

(I) For each $P \in \mathcal{P}, P \rightsquigarrow R$.

(II) For all planar surfaces $Q$, if $P \rightsquigarrow Q$ for all $P \in \mathcal{P}$, then $R \rightsquigarrow Q$.

We call $R$ the fusion of the surfaces in $\mathcal{P}$, and use notation for $R$ as described under the statement of Theorem 19 .

We note that $\tilde{\mathcal{M}}$ is by definition the (set-theoretic) moduli space of trivial structures on the pointed disk $\left(\Delta, x_{0}\right)$. As such $\tilde{\mathcal{M}}$ is a subset of the (set-theoretic) moduli space of trivial structures on all pointed surfaces as defined in \$5.1. Therefore, we can deduce our original Fusion Theorem (Theorem 19) from the Generalized Fusion Theorem using the following.

Proposition 22. If $\mathcal{P}$ is a collection of trivial surfaces homeomorphic to disks, then so is $\curlyvee \mathcal{P}$.

Proof. Suppose to the contrary that $R=\curlyvee \mathcal{P}$ is not simply connected. Let $\tilde{R}$ be the universal cover of $R$ and let $\pi: \tilde{R} \rightarrow R$ be the covering. We will prove that $\tilde{R}$ also satisfies statements (I) and (II) of the Fusion Theorem, contradicting uniqueness unless $\tilde{R}=R$.

Since each $P \in \mathcal{P}$ is simply connected, the immersion $P \rightsquigarrow R$ lifts to an immersions $P \rightsquigarrow \tilde{R}$. Thus (I) is satisfied by $\tilde{R}$. Now suppose that $P \rightsquigarrow S$ for all $P \in \mathcal{P}$. By statement (II) for $R$, we know $R \rightsquigarrow S$. The covering map $\tilde{R} \rightarrow R$ is an immersion, so by composition with the covering map, $\tilde{R} \rightsquigarrow S$. Thus, (II) is satisfied.

For the rest of the section we will work on proving the Generalized Fusion Theorem, and we will only study trivial surfaces. We prove this theorem in a series of steps starting with uniqueness (assuming existence).

Proof of uniqueness in the Generalized Fusion Theorem. Suppose there are two trivial surfaces $R_{1}$ and $R_{2}$ which satisfy statements (I) and (II) of the theorem. Then by statement (I), for each $j \in\{1,2\}$ and each $P \in \mathcal{P}$, we have $P \rightsquigarrow R_{j}$. Then by statement (II), we have $R_{1} \rightsquigarrow R_{2}$ and $R_{2} \rightsquigarrow R_{1}$. So $R_{1}=R_{2}$ by Corollary 9 , which may be seen to hold for trivial surfaces. (The same proof works as for planar surfaces.) 
5.3. Construction of the fusion. We construct the fusion of $\mathcal{P}$ as a quotient of the disjoint union $\bigsqcup_{P \in \mathcal{P}} P$. We make this a topological space by making each open set in any $P \in \mathcal{P}$ open in the disjoint union.

The bundle-wide developing map Dev is defined on the union of all trivial surfaces, so by restriction, it is also defined on the disjoint union $\bigsqcup_{P \in \mathcal{P}} P$.

Let $\sim$ be an equivalence relation on $\bigsqcup_{P \in \mathcal{P}} P$. Make the following choices:

- Let $P_{1}$ and $P_{2}$ be a pair of (not necessarily distinct) surfaces in $\mathcal{P}$.

- Let $r_{1} \in P_{1}$ and $r_{2} \in P_{2}$ be so that $r_{1} \sim r_{2}$.

- Let $\gamma_{1}:[0,1] \rightarrow P_{1}$ and $\gamma_{2}:[0,1] \rightarrow P_{2}$ be paths so that $\gamma_{1}(0)=r_{1}$, $\gamma_{2}(0)=r_{2}$ and

$$
\text { Devo } \gamma_{1}(t)=\operatorname{Dev} \circ \gamma_{2}(t) \text { for all } t \in[0,1] .
$$

We say that $\sim$ is path invariant if for every choice made as above, we have $\gamma_{1}(1) \sim$ $\gamma_{2}(1)$.

Theorem 23 (Constructive Fusion Theorem). Let $\sim$ be the smallest path invariant equivalence relation on $\bigsqcup_{P \in \mathcal{P}} P$ so that for each pair of surfaces $P$ and $Q$ in $\mathcal{P}$, we have $o_{P} \sim o_{Q}$. Then, $\bigsqcup_{P \in \mathcal{P}} P / \sim$ is isomorphic to the fusion of $\mathcal{P}$. Here, the local homeomorphism from $\bigsqcup_{P \in \mathcal{P}} P / \sim$ to $\mathbb{R}^{2}$ is provided by the developing map, which descends to this quotient.

We establish a corollary to the Constructive Fusion Theorem, which reveals some structure of the fusion of infinitely many surfaces which is unapparent in the original Fusion Theorem.

Corollary 24. Let $\mathcal{P}$ be an infinite collection of trivial surfaces. Let $R=\curlyvee \mathcal{P}$. For $P \in \mathcal{P}$, let $\iota_{P}: P \rightsquigarrow R$ be the immersion guaranteed by statement (I) of the Fusion Theorem. Let $p$ be a point in $P \in \mathcal{P}$, and let $q$ be a point in $Q \in \mathcal{P}$, and suppose that $\iota_{P}(p)=\iota_{Q}(q)$. Then there is a finite subcollection $\mathcal{F} \subset \mathcal{P}$ containing $P$ and $Q$ so that the immersions $j_{P}: P \rightsquigarrow \bigvee \mathcal{F}$ and $j_{Q}: Q \rightsquigarrow \bigvee \mathcal{F}$ satisfy $j_{P}(p)=j_{Q}(q)$.

Proof. Let $\sim$ be the equivalence relation from the Constructive Fusion Theorem. We think of equivalence relations on $\bigsqcup_{P \in \mathcal{P}} P$ as subsets of $\left(\bigsqcup_{P \in \mathcal{P}} P\right)^{2}$. We will construct an increasing sequence of equivalence relations $\sim_{n}$ on $\bigsqcup_{P \in \mathcal{P}} P$ so that $\bigcup_{n} \sim_{n}=\sim$. Then, the finiteness result follows if the finiteness result is proved for each $\sim_{n}$.

We define $\sim_{n}$ inductively in the integers $n \geq 0$ beginning with $\sim_{0}$. Let $p, q \in$ $\bigsqcup_{P \in \mathcal{P}} P$. We define $p \sim_{0} q$ if $p=q$ or if $p$ and $q$ are both basepoints of surfaces in $\mathcal{P}$. This can be seen to be an equivalence relation. Now suppose that $\sim_{n}$ is defined and let $p \in P \in \mathcal{P}$ and $q \in Q \in \mathcal{P}$ be points in $\bigsqcup_{P \in \mathcal{P}} P$. We say $p$ is $n+1$-related to $q\left(\right.$ denoted $\left.p \equiv_{n+1} q\right)$ if there are curves $\gamma_{1}:[0,1] \rightarrow P$ and $\gamma_{2}:[0,1] \rightarrow Q$ so that $\gamma_{1}(0) \sim_{n} \gamma_{2}(0), \gamma_{1}(1)=p, \gamma_{2}(1)=q$ and Dev $\circ \gamma_{1}(t)=$ Dev $\circ \gamma_{2}(t)$ for all $t \in[0,1]$. Observe that $\sim_{n} \subset \equiv_{n+1}$. We define $\sim_{n+1}$ to be the smallest equivalence relation containing $\equiv_{n+1}$. Since $\equiv_{n+1}$ is reflexive and symmetric, we can concretely say that $p \sim_{n+1} q$ if $p \equiv_{n+1} q$ or if there is a finite collection $p_{1}, p_{2}, \ldots, p_{k} \in \bigsqcup_{P \in \mathcal{P}} P$ so that the following holds:

$$
p \equiv_{n+1} p_{1} \equiv_{n+1} p_{2} \equiv_{n+1} \ldots \equiv_{n+1} p_{k} \equiv_{n+1} q .
$$

Observe that by definition of $\sim$ we have $\bigcup_{n} \sim_{n}=\sim$.

We now prove our finiteness statement by induction. Let $p \in P \in \mathcal{P}$ and $q \in Q \in \mathcal{P}$. If $p$ and $q$ are the same point, then $P=Q$, we can take $\mathcal{F}=\{P\}$ so 
that $P=\curlyvee \mathcal{F}$, and the identity map $P \rightsquigarrow P$ sends $p$ and $q$ to the same point. If $p$ and $q$ are basepoints of $P$ and $Q$, respectively, then the immersions $P \rightsquigarrow(P \curlyvee Q)$ and $Q \rightsquigarrow(P \curlyvee Q)$ carry these points to the basepoint of $P \curlyvee Q$ by definition of immersion. This proves the finiteness statement for $\sim_{0}$.

Now suppose $\sim_{n}$ satisfies the finiteness statement, and suppose that $p \equiv_{n+1} q$. Then, there must be curves $\gamma_{1}:[0,1] \rightarrow P$ and $\gamma_{2}:[0,1] \rightarrow Q$ as above. Then $\gamma_{1}(0) \sim_{n} \gamma_{2}(0)$, so there is a finite collection $\mathcal{F} \subset \mathcal{P}$ containing $P$ and $Q$ so that the immersions $\iota_{P}: P \rightsquigarrow \mathcal{F}$ and $\iota_{Q}: P \rightsquigarrow \mathcal{F}$ satisfy $\iota_{P} \circ \gamma_{1}(0)=\iota_{Q} \circ \gamma_{2}(0)$. Then by the Constructive Fusion Theorem applied to $\mathcal{Y} \mathcal{F}$, we see that $\iota_{P} \circ \gamma_{1}(1)=\iota_{Q} \circ \gamma_{2}(1)$ as well. This proves the finiteness statement for $\equiv_{n+1}$.

Now suppose that $\equiv_{n+1}$ satisfies the finiteness statement, and suppose that $p \sim_{n+1} q$. Then there are points $p_{1}, p_{2}, \ldots, p_{k} \in \bigsqcup_{P \in \mathcal{P}} P$ satisfying equation (6). Let $p_{0}=p$ and $p_{k+1}=q$. Let $P_{j} \in \mathcal{P}$ be the surface containing $p_{j}$ for each $j$. Then for all $j \in\{0, \ldots, k\}$, there is a finite collection $\mathcal{F}_{j}$ so that the immersions $\iota_{j}: P_{j} \rightsquigarrow \bigvee \mathcal{F}_{j}$ and $\iota_{j}^{\prime}: P_{j+1} \rightsquigarrow \bigvee \mathcal{F}_{j}$ satisfy $\iota_{j}\left(p_{j}\right)=\iota_{j}^{\prime}\left(p_{j+1}\right)$. Let $\mathcal{F}=\bigcup_{j=0}^{k} \mathcal{F}_{j}$. Then, there are immersions $\jmath_{j}: \bigvee \mathcal{F}_{j} \rightsquigarrow \bigvee \mathcal{F}$ for all $j$. The immersions $P_{j} \rightsquigarrow \bigvee \mathcal{F}$ can be given by $\jmath_{j} \circ \iota_{j}$ for $j \leq k$ and by $\jmath_{j-1} \circ \iota_{j-1}^{\prime}$ for $j \geq 1$. It follows that the image of $p_{j}$ inside $\bigvee \mathcal{F}$ is independent of $j \in\{0, \ldots, k+1\}$.

5.4. The fusion is a trivial surface. We will begin by proving that the quotient space described in the Constructive Fusion Theorem is really a trivial surface.

Lemma 25. Let $\sim$ be the equivalence relation from the Constructive Fusion Theorem. Then, the quotient $\bigsqcup_{P \in \mathcal{P}} P / \sim$ has the structure of a trivial surface with the associated immersion to $\mathbb{R}^{2}$ given by

$$
\phi([r])=\operatorname{Dev}(r) \quad \text { for all } r \in P \in \mathcal{P} .
$$

We will devote the remainder of the section to the proof of this fact. We now describe our plan. We will check that $\phi$ is a well-defined map. Then we will prove that $\phi$ is a local homeomorphism. This demonstrates that $\bigsqcup_{P \in \mathcal{P}} P / \sim$ is locally modeled on $\mathbb{R}^{2}$. Finally, we will show that $\bigsqcup_{P \in \mathcal{P}} P / \sim$ is Hausdorff. This proves that it is a surface, and $\phi$ gives this surface a trivial planar structure.

Proof that $\phi$ is well-defined. The basepoint of $\bigsqcup_{P \in \mathcal{P}} P / \sim$ is given by the equivalence class $\left[o_{P}\right]$ for some (any) $P \in \mathcal{P}$. We note that the developing map sends $o_{P}$ to zero. By induction, we can see that the points we are forced to identify by path invariance (see the definition above Theorem 23) also have the same image under the developing map. Therefore, $\phi$ is well-defined.

In order to prove the remainder of the lemma, it is useful to use the following.

Proposition 26. Let $r_{1} \in P_{1}$ for some $P_{1} \in \mathcal{P}$. Let $B \subset P_{1}$ be an open metric ball of radius $\epsilon$ about $r_{1}$ with $\epsilon$ taken to be so small that the developing map restricted to $B$ gives a homeomorphism to a ball of radius $\epsilon$ in the plane. Let

$$
U=\left\{r_{2} \in \bigsqcup_{P \in \mathcal{P}} P: r_{2} \sim b \text { for some } b \in B\right\}
$$

Then, $U$ is open in $\bigsqcup_{P \in \mathcal{P}} P$, and so by definition $B^{\prime}=\{[b]: b \in B\}$ is open in $\bigsqcup_{P \in \mathcal{P}} P / \sim$. 
Proof. We remind the reader of the topology we placed on $\bigsqcup_{P \in \mathcal{P}} P$. We need to show that $U \cap P_{2}$ is open in $P_{2}$ for all $P_{2} \in \mathcal{P}$. Let $r_{2} \in U \cap P_{2}$. Then, $r_{2} \sim b$ for some $b \in B$. Since the image under the developing map is an $\sim$-invariant, we have

$$
\left|\operatorname{Dev}\left(r_{1}\right)-\operatorname{Dev}\left(r_{2}\right)\right|=\left|\operatorname{Dev}\left(r_{1}\right)-\operatorname{Dev}(b)\right|<\epsilon,
$$

because $b$ lies in the ball $B$ of radius $\epsilon$ about $r_{1}$. Choose $\epsilon^{\prime}>0$ small enough so that the following hold:

(1) The developing map restricted to the open $\epsilon^{\prime}$-ball about $r_{2}$ is a homeomorphism to a ball in the plane of radius $\epsilon^{\prime}$.

(2) The $\epsilon^{\prime}$-ball about $b$ is a subset of the ball $B$.

Let $D$ denote the open ball of radius $\epsilon^{\prime}$ about $r_{2}$ in $P_{2}$. Let $r_{3} \in D$. Then by (1) there is a path $\gamma_{1}$ of length less than $\epsilon^{\prime}$ joining $r_{2}$ to $r_{3}$. Similarly, there is a path $\gamma_{2}$ starting at $b$ and contained in $B$ so that Dev $\circ \gamma_{1}=D e v \circ \gamma_{2}$. This path stays within $B$ by (2). Thus $U \cap P_{2}$ is open as desired.

Proof that $\phi$ is a local homeomorphism. Choose any $\left[r_{1}\right] \in \bigsqcup_{P \in \mathcal{P}} P / \sim$, and choose $r_{1} \in\left[r_{1}\right]$. Let $U$ be as in the above proposition. Then the set $B^{\prime}$ at the end of the proposition is an open set containing $\left[r_{1}\right]$. Furthermore, since each point in $B^{\prime}$ has a unique representative in $B$, we know that $\left.\phi\right|_{B^{\prime}}$ is one-to-one and onto its image, which is an open ball in $\mathbb{R}^{2}$.

We must prove that $\left.\phi\right|_{B^{\prime}}$ is continuous. This also follows from the proposition. Let $\mathbf{v}$ be a point in $\phi\left(B^{\prime}\right)$, and let $\left[r_{2}\right]=\phi^{-1}(\mathbf{v})$. We can choose the representative $r_{2} \in\left[r_{2}\right] \cap B$. Then the neighborhood of radius $\epsilon-\left|\operatorname{Dev}\left(r_{1}\right)-\operatorname{Dev}\left(r_{2}\right)\right|$ about $r_{2}$ is isometric to a Euclidean ball. Applying the proposition to this choice of center $r_{2}$ and radius produces an open set containing $r_{2}$ and contained in $B^{\prime}$.

The fact that $\left(\left.\phi\right|_{B^{\prime}}\right)^{-1}$ is continuous is a tautology, because of the topology we placed on $\bigsqcup_{P \in \mathcal{P}} P$. Recall that the union of equivalence classes in $B^{\prime}$ is open. Call this union $U$ as in the lemma above. Now let $C^{\prime} \subset B^{\prime}$ be a smaller open set. This by definition means that its union of equivalence classes $V^{\prime} \subset U^{\prime}$ is open. That is, $V^{\prime} \cap P$ is open for each $P \in \mathcal{P}$. Moreover by definition of $\phi$, we have

$$
\left.\phi\right|_{B^{\prime}}\left(C^{\prime}\right)=\operatorname{Dev}\left(V^{\prime}\right)=\left.\bigcup_{P \in \mathcal{P}} \operatorname{Dev}\right|_{P}\left(V^{\prime} \cap P\right) .
$$

But, the image of any open set in a trivial surface under the developing map is open in $\mathbb{R}^{2}$, and any union of open sets is open.

Proof that $\bigsqcup_{P \in \mathcal{P}} P / \sim$ is Hausdorff. Let $\left[r_{1}\right],\left[r_{2}\right] \in \bigsqcup_{P \in \mathcal{P}} P / \sim$ be distinct. We will separate these points by open sets. First suppose that $\phi\left(\left[r_{1}\right]\right) \neq \phi\left(\left[r_{2}\right]\right)$. Then by constructing neighborhoods around each of $\left[r_{1}\right]$ and $\left[r_{2}\right]$ using Proposition 26 with radius less than or equal to $\frac{1}{2}\left|\phi\left(\left[r_{1}\right]\right)-\phi\left(\left[r_{2}\right]\right)\right|$ produces open sets which can be discerned to be disjoint because their images under $\phi$ are disjoint.

Now suppose that $\left[r_{1}\right]$ and $\left[r_{2}\right]$ are distinct but that $\phi\left(\left[r_{1}\right]\right)=\phi\left(\left[r_{2}\right]\right)$. Choose representatives $r_{1} \in\left[r_{1}\right]$ and $r_{2} \in\left[r_{2}\right]$. Suppose $r_{1} \in P_{1}$ and $r_{2} \in P_{2}$. Let $B_{1} \subset P_{1}$ and $B_{2} \subset P_{2}$ be open metric balls about $r_{1}$ and $r_{2}$, respectively, which are each isometric to a Euclidean metric ball. These balls determine open sets $B_{1}^{\prime}, B_{2}^{\prime} \subset \bigsqcup_{P \in \mathcal{P}} P / \sim$ by Proposition 26. We claim that they are disjoint. Suppose not. Then, there is an $\left[r_{3}\right] \in B_{1}^{\prime} \cap B_{2}^{\prime}$. By the proposition, we can then find points $b_{1} \in B_{1} \cap\left[r_{3}\right]$ and $b_{2} \in B_{2} \cap\left[r_{3}\right]$. Since $b_{1} \sim b_{2}$, they have the same image under Dev. Parameterize the line segments joining $b_{1}$ to $r_{1}$ within $B_{1}$ and joining $b_{2}$ to $r_{2}$ 
within $B_{2}$ in the same way. Then, path invariance guarantees that $r_{1} \sim r_{2}$. This contradicts the distinctness of $\left[r_{1}\right]$ and $\left[r_{2}\right]$.

5.5. Proof of the Fusion Theorem. We now prove the Constructive Fusion Theorem. Note that this immediately implies the Generalized Fusion Theorem (Theorem 21), and the original version of the Fusion Theorem (Theorem 19) follows from Proposition 22

Proof of Theorem 23. Let $P \in \mathcal{P}$. Let $R=\bigsqcup_{P \in \mathcal{P}} P / \sim$, where $\sim$ is the equivalence relation described in the theorem. We will prove that $R$ has the properties described in the Fusion Theorem.

Statement (I) of Theorem 21 simply requires proving that the natural maps $P \rightarrow R$ respect the basepoints, respect the developing maps, and are local homeomorphisms. Basepoints are respected by construction. By definition of $\phi$, the developing map is respected. This proves that the natural map $P \rightarrow R$ is an immersion. Finally, the fact that $P \rightarrow R$ is a local homeomorphism follows from the fact that the developing maps are respected and are local homeomorphisms.

Statement (II) of Theorem 21 reduces to a statement about equivalence relations. Suppose $P \rightsquigarrow S$ for all $P \in \mathcal{P}$. Let $j: \bigsqcup_{P \in \mathcal{P}} P \rightarrow S$ be the simultaneous immersion of all planar surfaces $P \in \mathcal{P}$ into $S$. Then, we define an equivalence relation on $\bigsqcup_{P \in \mathcal{P}} P$ by $p \approx q$ for $p \in P \in \mathcal{P}$ and $q \in Q \in \mathcal{P}$ if $j(p)=j(q)$. Then all basepoints are equivalent and $\approx$ is path invariant. Since $\sim$ is the smallest such relation, each $\sim$-equivalence class is contained in an $\approx$-equivalence class. This gives a canonical map $R \rightarrow S$. By construction, it is an immersion.

\section{NEW OPEN SETS AND SECOND-COUNTABILITY}

In this section, we will study subsets of planar surfaces which are unions of rectangles. Analyzing such sets will enable us to prove two important results about the immersive topology.

Theorem 27 (Open sets in $\tilde{\mathcal{M}})$. Let $P$ be a planar surface. If $K \in P C(P)$ is compact, then both $\tilde{\mathcal{M}}_{\rightsquigarrow}(K)$ and $\tilde{\mathcal{M}}_{\hookrightarrow}(K)$ are open in the immersive topology on $\tilde{\mathcal{M}}$. If $U \in P C(P)$ is open, then both $\tilde{\mathcal{M}}_{\varkappa}(U)$ and $\tilde{\mathcal{M}}_{\hookrightarrow \rightarrow}(U)$ are open.

Theorem 28. The immersive topologies on $\tilde{\mathcal{M}}$ and $\tilde{\mathcal{E}}$ are second-countable, that is, they admit a countable basis.

We remark that Propositions 38 and 39 will give explicit countable subbases for these topologies. (By general principles, the induced bases are then also countable.)

6.1. Definition of rectangular union. A closed rectangle in the plane is a subset of $\mathbb{R}^{2}$ of the form

$$
[a, b] \times[c, d]=\left\{(x, y) \in \mathbb{R}^{2}: a \leq x \leq b \text { and } c \leq y \leq d\right\},
$$

where $a<b$ and $c<d$. Similarly, an open rectangle is a set of the form $(a, b) \times(c, d)$. We call such a rectangle rational if $a, b, c$, and $d$ are rational numbers.

Let $P$ be a planar surface. We call $R \subset P$ a closed (resp., open) rectangle if $\operatorname{Dev}(R)$ is a closed (resp., open) rectangle and the restriction $\left.\operatorname{Dev}\right|_{R}: R \rightarrow \operatorname{Dev}(R)$ is a homeomorphism. We say $R$ is rational if $\operatorname{Dev}(R)$ is.

A closed (resp., open) rectangular union is a finite union of closed (resp., open) rectangles in a planar surface which is connected and whose boundary is a disjoint 
collection of curves. We call a rectangular union rational if it can be constructed as a finite union of rational rectangles.

Proposition 29. The closure of an open rectangular union with compact closure is a closed rectangular union. The interior of a closed rectangular union is an open rectangular union.

Proof. Let $P$ be a planar surface. Suppose $\left\{R_{i} \subset P\right\}$ is a finite collection of open rectangles whose union is an open rectangular union $U$ with compact closure. Then $\bar{U}=\bigcup_{i} \bar{R}_{i}$ is a closed rectangular union.

Now suppose $\mathcal{R}=\left\{R_{i} \subset P\right\}$ is a collection of closed rectangles whose union is a closed rectangular union $K$. Then $\bigcup_{i} R_{i}^{\circ}$ may not be as large as $K^{\circ}$. We will construct a larger finite collection of closed rectangles $\mathcal{R}^{\prime} \supset \mathcal{R}$ so that $K^{\circ}=$ $\bigcup_{R \in \mathcal{R}^{\prime}} R^{\circ}$. We will describe an algorithm for constructing $\mathcal{R}^{\prime}$ by adding rectangles beginning with $\mathcal{R}^{\prime}=\mathcal{R}$. Let $\Lambda=K^{\circ} \backslash \bigcup_{i} R_{i}^{\circ}$. A point $p \in \Lambda$ is either a vertex of a rectangle in $\mathcal{R}$ or lies in the interior of an edge of such a rectangle. If $p \in \Lambda$ is a vertex, then it has a neighborhood which lies in $K^{\circ}$, so we can add a small rectangle to $\mathcal{R}$ which contains $p$ and is contained entirely in $K^{\circ}$. We add such a rectangle to $\mathcal{R}^{\prime}$ for each vertex in $\Lambda$. Now suppose $p \in \Lambda$ is not a vertex. Then, it must lie in the common boundary of two rectangles $R_{1}$ and $R_{2}$ lying on opposite sides of a line whose edges intersect in an interval. We can construct a closed rectangle, $R_{0}$, which is contained in $R_{1} \cup R_{2}$ and contains the overlap $R_{1} \cap R_{2}$. See below:

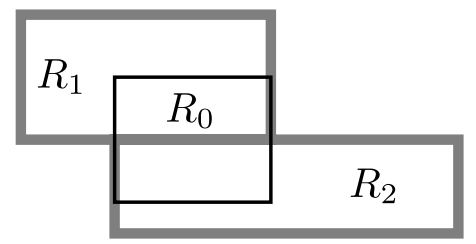

We add such a rectangle to $\mathcal{R}^{\prime}$ for all pairs of rectangles in $\mathcal{R}$ which intersect in an interval. The resulting $\mathcal{R}^{\prime}$ has the desired property.

Proposition 30. An open rectangular union is homeomorphic to a finitely punctured disk.

Proof. By definition, an open rectangular union is a connected surface in a topological disk. So, it is homeomorphic to a punctured disk, but the number of punctures may be infinite. So, it suffices to prove that an open rectangular union has finite Euler characteristic.

We will show that a union of open rectangles, i.e., an arbitrary union of open rectangles in a planar surface, has finite Euler characteristic. We define the complexity of such a union to be the smallest number of rectangles necessary to write the set as a union of rectangles. In fact, we will prove that if a union of rectangles $U$ has complexity less than $n$, then $|\chi(U)|<2^{n}$. For $n=0, \chi(U)=0$, and for $n=1, \chi(U)=1$. These provide a base case, and we proceed by induction in $n$. Suppose the statement $|\chi(U)|<2^{n}$ for all $U$ of complexity $n$. Let $U^{\prime}$ be a union of complexity $n+1$. Then $U^{\prime}=U \cup R$, where $U$ has complexity $n$ and $R$ is another open rectangle. By the inclusion-exclusion principle,

$$
\chi(U \cup R)=\chi(U)+\chi(R)-\chi(U \cap R)=\chi(U)-\chi(U \cap R)+1 .
$$


Note that the intersection of two open rectangles in a planar surface is either empty or another open rectangle. In particular, $U \cap R$ is either empty or a union of rectangles of complexity no more than $n$. By inductive hypothesis, $|\chi(U)|<2^{n}$ and $|\chi(U \cap R)|<2^{n}$. So by equation (7),

$$
|\chi(U \cup R)| \leq|\chi(U)|+|\chi(U \cap R)|+1 \leq\left(2^{n}-1\right)+\left(2^{n}-1\right)+1<2^{n+1} .
$$

6.2. A finiteness condition. Let $P$ and $Q$ be planar surfaces. Let $A \in \operatorname{PC}(P)$ and $B \in \mathrm{PC}(Q)$. We say $A$ and $B$ are isomorphic if $A \rightsquigarrow B$ and $B \rightsquigarrow A$. This defines an equivalence relation on

$$
\mathrm{PC}=\bigcup_{P \in \tilde{\mathcal{M}}} \mathrm{PC}(P) .
$$

We note that sets of the form $\tilde{\mathcal{M}}_{\rightsquigarrow}(K), \tilde{\mathcal{M}}_{\hookrightarrow}(K), \tilde{\mathcal{M}}_{\varkappa}(U)$, and $\tilde{\mathcal{M}}_{\hookrightarrow \rightarrow}(U)$ only depend on the isomorphism classes of $K$ and $U$.

We state our main finiteness result for convex sets rather than rectangles because it will be useful in later sections.

Proposition 31. Let $A_{1}, \ldots, A_{n}$ be a collection of convex subsets of the plane which are either all open or all closed. There are only finitely many isomorphism classes of $U \in P C$ which are the union of sets of the form $\tilde{A}_{i} \subset U$ so that $A_{i}=\operatorname{Dev}\left(\tilde{A}_{i}\right)$ for all $i$.

The reason this proposition will be useful is that our topology on $\tilde{\mathcal{M}}$ was defined in terms of a subbasis, and finite intersections of elements of the subbasis are still open but typically not elements of the subbasis. We will eventually be taking intersections of sets of the form $\tilde{\mathcal{M}}_{\rightsquigarrow}(K)$ and $\tilde{\mathcal{M}}_{\hookrightarrow}(U)$ over finite collections of isomorphism classes in order to prove Theorem 27

Proof. Fix $A_{1}, \ldots, A_{n}$. Let $U=\bigcup_{i=1}^{n} \tilde{A}_{i}$ be such a union. Suppose $U$ lives in the planar surface $P$ with basepoint $o_{P}$. We associate $U$ to two pieces of information. First there is a subset $\mathcal{S}(U) \subset\{1, \ldots, n\}$ consisting of those $i$ so that $o_{P} \in \tilde{A}_{i}$. Also, we can associate to $U$ a subgraph $\mathcal{G}(U)$ of the complete graph $K_{n}$ with vertex set $\{1, \ldots, n\}$. We define this subgraph by the condition that there is an edge between distinct $i, j \in\{1, \ldots, n\}$ if $\tilde{A}_{i} \cap \tilde{A}_{j} \neq \emptyset$. Assume this intersection is non-empty. By convexity of $A_{i}$ and $A_{j}$, it follows that $A_{i} \cap A_{j}$ is convex and so connected. Because the lifts $\left.D e v\right|_{A_{i}} ^{-1}: A_{i} \rightarrow P$ and $\left.D e v\right|_{A_{j}} ^{-1}: A_{j} \rightarrow P$ agree at one point, they must agree at all points of $A_{i} \cap A_{j}$ by analytic continuation. Therefore, we can recover $U$ up to isomorphism from $\mathcal{S}(U)$ and $\mathcal{G}(U)$. Consider the disjoint union $\bigsqcup_{i} A_{i}$. Inclusion of each $A_{i}$ into $\mathbb{R}^{2}$ gives a natural map $\pi: \bigsqcup_{i} A_{i} \rightarrow \mathbb{R}^{2}$. Define the equivalence relation $\sim$ on the disjoint union by $p \in A_{i}$ is equivalent to $q \in A_{j}$ if $\pi(p)=\pi(q)$ and the edge $\overline{i j}$ lies in $\mathcal{G}(U)$. There is a natural identification between $U$ and $\bigsqcup_{i} A_{i} / \sim$ which picks out the isomorphism class of $U$. The collection of points $p \in A_{i}$ with $i \in \mathcal{S}(U)$ and $\operatorname{Dev}(p)=\mathbf{0}$ is an equivalence class of $\bigsqcup_{i} A_{i} / \sim$. This corresponds to the basepoint of $U$.

Let $U_{1}$ and $U_{2}$ be unions coming from the same choices of convex sets $A_{1}, \ldots, A_{n}$. We remark that there is an immersion $\iota: U_{1} \rightarrow U_{2}$ if and only if $\mathcal{S}\left(U_{1}\right) \subset \mathcal{S}\left(U_{2}\right)$ and $\mathcal{G}\left(U_{1}\right)$ is a subgraph of $\mathcal{G}\left(U_{2}\right)$. Viewing

$$
U_{1}=\bigsqcup_{i} A_{i} / \sim_{1} \quad \text { and } \quad U_{2}=\bigsqcup_{i} A_{i} / \sim_{2}
$$


we observe that these conditions imply that the identity map $\bigsqcup_{i} A_{i} \rightarrow \bigsqcup_{i} A_{i}$ induces a well-defined map from $U_{1} \rightarrow U_{2}$. This is the needed immersion.

It follows that the collection of unions satisfying the proposition is finite: There are no more than the number of pairs $(\mathcal{S}(U), \mathcal{G}(U))$, where $\mathcal{S}(U) \subset\{1, \ldots, n\}$ and $\mathcal{G}(U)$ is a subgraph of $K_{n}$.

Corollary 32. Let $U$ be an open (resp., closed) subset of a planar surface which is a finite union of open (resp., closed) convex sets. Then there are only finitely many images of $U$ under immersions up to isomorphism.

Proof. Let $U=\bigcup_{i=1}^{n} \tilde{A}_{i}$ be a union of convex sets in a planar surface $P$. Let $A_{i}=\operatorname{Dev}\left(\tilde{A}_{i}\right)$. Given an immersion $\iota: U \rightsquigarrow Q$, we have $\iota(U)=\bigcup_{i=1}^{n} \iota\left(\tilde{A}_{i}\right)$. This writes $\iota(U)$ as a union of lifts of the sets $A_{i} \subset \mathbb{R}^{2}$ for $i=1, \ldots, n$. There are only finitely many possibilities for $\iota(U)$ by Proposition 31

Corollary 33. There are only countably many isomorphism classes of (open or closed) rational rectangular unions in $P C$.

Proof. This follows from the proposition above, because there are only countably many finite collections of rational rectangles in the plane.

6.3. Rectilinear curves. We will say a closed curve $\gamma: \mathbb{R} / L \mathbb{Z} \rightarrow \mathbb{R}^{2}$ is rectilinear if there are $0=t_{0}<t_{1}<\ldots<t_{2 k}=L$ so that the derivative satisfies

$$
\gamma^{\prime}(t)= \begin{cases}( \pm 1,0) & \text { if } t_{j}<t<t_{j+1} \text { with } j \text { even } \\ (0, \pm 1) & \text { if } t_{j}<t<t_{j+1} \text { with } j \text { odd. }\end{cases}
$$

We say the rectilinear curve $\gamma$ is rational if the points $\gamma\left(t_{j}\right)$ are rational.

Our topology on $\tilde{\mathcal{M}}$ was defined using a subbasis consisting of elements of the form $\tilde{\mathcal{M}}_{\rightsquigarrow}(K)$ and $\tilde{\mathcal{M}}_{\hookrightarrow}(U)$ where $K$ and $U$ are disks, and so we will need to study rectangular unions which are also topological disks. Such disks are bounded by rectilinear curves, and we will use an understanding of rectilinear curves to deduce certain finiteness results.

Lemma 34. Let $\gamma$ be a closed immersed rectilinear curve in $\mathbb{R}^{2}$. Then, lifts of $\gamma$ to simple closed curves in planar surfaces bound at most finitely many isomorphism classes of disks, and each such disk is a rectangular union. Furthermore, if $\gamma$ is rational, then so is each rectangular union.

Proof. Consider the rectilinear curve $\gamma$ in $\mathbb{R}^{2}$. If it bounds an immersed disk, then $\gamma$ can be oriented so that the winding number around any point in the plane is non-negative. By rectilinearity, we can divide the bounded components of $\mathbb{R}^{2} \backslash \gamma$ into rectangles. Furthermore, if $\gamma$ is rational, these rectangles can be chosen all to be rational. Let $\mathcal{R}$ be the collection of such closed rectangles with multiplicity corresponding to the winding number.

Each immersed disk bounded by $\gamma$ can be assembled by identifying boundary edges of rectangles in $\mathcal{R}$. In particular by Proposition 31, there are only finitely many immersed disks with boundary $\gamma$. Furthermore, from this construction we see that each such disk is a rectangular union. If $\gamma$ was rational, then the disk is a rational rectangular union.

Corollary 35. Let $P$ be a planar surface and let $K \in P C(P)$ be a closed rectangular union. Then, there is a smallest $D \in \overline{D i s k}(P)$ so that $K \subset D$. Furthermore, $D$ is a rectangular union. 
Proof. By definition of rectangular union, $\partial K$ is a union of disjoint simple closed curves, each of which bounds a disk in $P$. Let $A$ be the unique unbounded component of $P \backslash K$. Then $A$ can only touch one boundary component of $P$, so $A$ is homeomorphic to an annulus, and $\partial A \subset P$ consists of this one component. The developed image $\operatorname{Dev}(\partial A)$ is a rectilinear curve. So, it bounds a rectangular union in $\overline{\operatorname{Disk}}(P)$ by the lemma above.

If $K \in \mathrm{PC}(P)$ is a closed rectangular union, then we call the disk $D$ provided by the corollary the smallest closed disk containing $K$. Similarly, any open set $U$ in a planar surface which is homeomorphic to a finitely punctured disk is contained in a smallest open disk obtained by filling in the compact components of the compliment.

Corollary 36. Let $P$ be a planar surface and $U \subset P$ be an open subset containing the basepoint and homeomorphic to a finitely punctured disk. If $Q \in \tilde{\mathcal{M}}$ and there is an embedding e $: U \hookrightarrow Q$, let $D(Q) \subset Q$ denote the smallest open disk containing $e(U)$. Then the set

$$
\{D(Q): Q \in \tilde{\mathcal{M}} \text { and } U \hookrightarrow Q\}
$$

contains only finitely many different isomorphism classes.

Proof. Orient the boundary components of $U$ so that traveling around $\partial U$ leaves $U$ on the left. For each boundary component choose a smooth simple closed curve in $U$ homotopic to the boundary component with the same orientation. This allows us to distinguish the boundary component which does not bound a compact subset of $P$, namely the one whose associated curve has turning number 1 . (The other curves have turning number -1 since they bound a disk with the wrong orientation.)

Now we can choose a closed curve $\tilde{\gamma}$ in $U$ which is homotopic to the distinguished boundary component so that $\gamma=\left.D e v\right|_{P} \circ \tilde{\gamma}$ is a rectilinear curve. Let $\mathcal{V} \subset \tilde{\mathcal{M}}$ denote the collection isomorphism classes of planar surfaces bounded by lifts of $\gamma$. The set $\mathcal{V}$ is finite by Lemma 34 .

Now suppose that $R$ is a planar surface and there is an embedding $e: U \hookrightarrow R$. Let $V$ be the open disk bounded by $e(\tilde{\gamma})$. The isomorphism class of $V$ lies in $\mathcal{V}$. The set $e(U) \cup V$ is the smallest open disk containing $e(U)$. Furthermore this disk is uniquely determined by the isomorphism class $V$. To see this suppose that $R^{\prime}$ is another planar surface, $e^{\prime}: U \hookrightarrow R^{\prime}$ is an embedding, $V^{\prime}$ is the open disk bounded by $e(\tilde{\gamma})$ and $V$ and $V^{\prime}$ are isomorphic. Let $f: V \rightarrow V^{\prime}$ denote this isomorphism. Define the map

$$
g: e(U) \cup V \rightarrow e^{\prime}(U) \cup V^{\prime} ; \quad x \mapsto \begin{cases}e^{\prime} \circ e^{-1}(x) & \text { if } x \in e(U), \\ f(x) & \text { if } x \in V .\end{cases}
$$

This map is ambiguous on $V \cap e(U)$, but this set is a path-connected open set and the maps $e^{\prime} \circ e^{-1}$ and $f$ restricted to this set are both embeddings so they agree. Furthermore, $g$ is an immersion because it is an immersion locally. We similarly get a map from $e^{\prime}(U) \cup V^{\prime} \rightarrow e(U) \cup V$, showing that these two sets are isomorphic. This proves that the isomorphism class of the smallest open disk containing an embedded image of $U$ is uniquely determined by $V$ as defined above and taken from the finite set $\mathcal{V}$.

\subsection{Constructing rectangular unions.}

Theorem 37. Let $P$ be a planar surface, and let $K_{1}, U \in P C(P)$ with $K_{1}$ compact, $U$ open and $K_{1} \subset U$. Then, there is a closed rational rectangular union $K_{2} \in P C(P)$ 
so that $K_{1} \subset K_{2}^{\circ}$ and $K_{2} \subset U$. Furthermore, if $U$ is homeomorphic to an open disk, then we can arrange that $K_{2} \in \overline{\operatorname{Disk}}(P)$.

Proof. We will deal with the last sentence later in the proof, for now we will produce a $K_{2} \in \mathrm{PC}(P)$. For every $p \in K_{1}$, choose a closed rational rectangle $R_{p}$ so that $p \in R_{p} \subset U$. Then $\left\{R_{p}^{\circ}: p \in K_{1}\right\}$ is an open cover of $K_{1}$. By compactness, there is a finite subcover. Let $\left\{R_{1}, \ldots, R_{n}\right\}$ be the corresponding collection of closed rectangles. Let $K_{2}=\bigcup_{i=1}^{n} R_{i}$. This set is path connected and contains the basepoint because $K_{1}$ does. Also by construction, we have $K_{1} \subset K_{2}^{\circ}$ and $K_{2} \subset U$.

It may not be true that $K_{2}$ is a rectangular union because $\partial K_{2}$ could fail to be bounded by disjoint curves. This can only happen if some rectangles share a common vertex but are situated diagonally across from each other as depicted below.
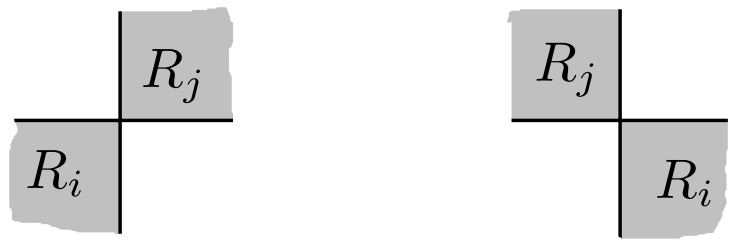

We can fix this problem by adding a rectangle centered at the common vertex which is small enough to be contained in $U$ and only intersect the edges of rectangles in the set $\left\{R_{1}, \ldots, R_{n}\right\}$ in edges which contain the common vertex.

Now suppose that $U$ is a topological disk. Replacing the $K_{2}$ constructed above by the smallest closed disk containing $K_{2}$ gives the last statement. See Corollary 35

6.5. Open sets in $\tilde{\mathcal{M}}$. In this subsection we prove Theorem 27, namely that sets of the form $\tilde{\mathcal{M}}_{\rightsquigarrow}(K), \tilde{\mathcal{M}}_{\hookrightarrow}(K), \tilde{\mathcal{M}}_{\hookrightarrow}(U)$, and $\tilde{\mathcal{M}}_{\hookrightarrow}(U)$ are open in $\tilde{\mathcal{M}}$.

Proof of Theorem 27. We prove Theorem 27 using the definition of the immersive topology. That is, we only assume sets of the form $\tilde{\mathcal{M}}_{\rightsquigarrow}(K)$ and $\tilde{\mathcal{M}}_{\hookrightarrow \rightarrow}(U)$ are open when $K$ and $U$ are closed and open topological disks, respectively.

Let $P$ be a planar surface, and let $K \in \mathrm{PC}(P)$ be compact. We will show that $\tilde{\mathcal{M}}_{\rightsquigarrow}(K)$ is open. Choose $Q \in \tilde{\mathcal{M}}_{\rightsquigarrow}(K)$. Then by definition, there is an immersion $\iota: K \rightsquigarrow Q$. Let $K_{1}=\iota(K)$. By choosing a closed disk family in $Q$, we can find a $K_{3} \in \overline{\operatorname{Disk}}(Q)$ so that $K_{1} \subset K_{3}^{\circ}$. Then Theorem 37guarantees that there is a closed rational rectangular union $K_{2} \in \overline{\operatorname{Disk}}(Q)$ so that $K_{1} \subset K_{2}^{\circ}$ and $K_{2} \subset K_{3}^{\circ}$. Since $\tilde{\mathcal{M}}_{\rightsquigarrow}\left(K_{2}\right)$ is open, it suffices to prove that $\tilde{\mathcal{M}}_{\rightsquigarrow}\left(K_{2}\right) \subset \tilde{\mathcal{M}}_{\rightsquigarrow}(K)$. Let $R \in \tilde{\mathcal{M}}_{\rightsquigarrow}\left(K_{2}\right)$. Then, there is an immersion $j: K_{2} \rightsquigarrow R$. The composition $j \circ \iota: K \rightsquigarrow R$ is the immersion needed to prove that $R \in \tilde{\mathcal{M}}_{\rightsquigarrow}(K)$.

Let $P$ be a planar surface, and let $U \in \mathrm{PC}(P)$ be open. We will show that $\tilde{\mathcal{M}}_{\hookrightarrow}(U)$ is open. Choose $Q \in \tilde{\mathcal{M}}_{\hookrightarrow}(U)$. Choose an exhaustion of $U$ by an increasing sequence of compact sets $D_{n} \in \mathrm{PC}(P)$ whose interiors $D_{n}^{\circ}$ lie in $\mathrm{PC}(P)$ so that each $D_{n} \subset D_{n+1}^{\circ}$. By Proposition 15, if each $D_{n}^{\circ}$ embeds in $Q$, then $U$ would embed in $Q$. So there is an $a>0$ so that $D_{a} \hookrightarrow Q$. By Theorem 37, there is a closed rectangular union $K_{2}$ so that $D_{a} \subset K_{2}^{\circ}$ and $K_{2} \subset D_{a+1}^{\circ}$. Because $D_{a}^{\circ} \subset K_{2}^{\circ} \subset U$, we have

$$
Q \in \tilde{\mathcal{M}}_{\hookrightarrow \rightarrow}\left(D_{a}^{\circ}\right) \subset \tilde{\mathcal{M}}_{\hookrightarrow \rightarrow}\left(K_{2}^{\circ}\right) \subset \tilde{\mathcal{M}}_{\hookrightarrow \rightarrow}(U) .
$$


It suffices to show that $\tilde{\mathcal{M}}_{\hookrightarrow \rightarrow}\left(K_{2}^{\circ}\right)$ is open. Let $\mathcal{D}$ be the collection of all isomorphism classes of smallest open disks containing embedded images of $K_{2}^{\circ}$. This set is finite by Proposition 30 together with Corollary 36. If $K_{2}^{\circ}$ embeds in a planar surface $R$, then there is an element $D \in \mathcal{D}$ which also embeds. It follows that

$$
\tilde{\mathcal{M}}_{\hookrightarrow \rightarrow}\left(K_{2}^{\circ}\right)=\bigcap_{D \in \mathcal{D}} \tilde{\mathcal{M}}_{\hookrightarrow \rightarrow}(D),
$$

which is open by definition of the topology.

Let $P$ be a planar surface and $U \in \mathrm{PC}(P)$ be open. We will now show that $\tilde{\mathcal{M}}_{\varkappa}(U)$ is open. Choose $Q \in \tilde{\mathcal{M}}_{\varkappa \hookrightarrow}(U)$. By the same reasoning as above, we can find an closed rectangular union $K_{2} \in \overline{\operatorname{Disk}}(P)$ so that $K_{2}^{\circ} \subset U$ and $K_{2}^{\circ} \not \rightarrow Q$. Furthermore, $\tilde{\mathcal{M}}_{\varkappa}\left(K_{2}^{\circ}\right) \subset \tilde{\mathcal{M}}_{\varkappa}(U)$. Let $\mathcal{V}$ be the collection of all immersed images of $K_{2}^{\circ}$. The set $\mathcal{V}$ is finite by Corollary [32, From the above paragraph, we know that $\tilde{\mathcal{M}}_{\hookrightarrow \rightarrow}(V)$ is open for every $V \in \mathcal{V}$. Thus,

$$
\tilde{\mathcal{M}}_{\not \nrightarrow}\left(K_{2}^{\circ}\right)=\bigcap_{V \in \mathcal{V}} \tilde{\mathcal{M}}_{\hookrightarrow \rightarrow}(V)
$$

is open.

Finally, let $P$ be a planar surface, and let $K \in \mathrm{PC}(P)$ be compact. We will show that $\tilde{\mathcal{M}}_{\hookrightarrow}(K)$ is open. Choose any $Q \in \tilde{\mathcal{M}}_{\hookrightarrow}(K)$. Then there is an embedding $e: K \hookrightarrow Q$. Let $K_{1}=e(K)$. Choose $K_{3} \in \overline{\operatorname{Disk}}(Q)$ so that $K_{1} \subset K_{3}^{\circ}$. Then we can find a $K_{2} \in \overline{\operatorname{Disk}}(Q)$ which is a closed rectangular union and satisfies $K_{1} \subset K_{2}^{\circ}$ and $K_{2} \subset K_{3}^{\circ}$. Let $\mathcal{L}$ be the collection of all immersed images of $K_{2}^{\circ}$ up to isomorphism. This collection is finite by Corollary 32, Let $\mathcal{L}_{0}$ be $\mathcal{L}$ with the equivalence class of $K_{2}^{\circ}$ itself removed. Then if $L \in \mathcal{L}_{0}$, the immersion $K_{2}^{\circ} \rightsquigarrow L$ is not an embedding. Let $R$ be a planar surface. Suppose that there is an immersion $\iota: K_{2} \rightsquigarrow R$ and that for each $L \in \mathcal{L}_{0}$, we have $L \hookrightarrow R$. Note that by restriction of $\iota$, we have $K_{2}^{\circ} \rightsquigarrow R$. Then by definition of $\mathcal{L}_{0}$, our immersion $K_{2}^{\circ} \rightsquigarrow R$ must actually be an embedding. So, by restriction, $\left.\iota\right|_{K_{1}}: K_{1} \hookrightarrow R$, and by composition $\iota \circ e: K \hookrightarrow R$. It follows that

$$
Q \in \tilde{\mathcal{M}}_{\rightsquigarrow}\left(K_{2}\right) \cap \bigcap_{L \in \mathcal{L}_{0}} \tilde{\mathcal{M}}_{\hookrightarrow}(L) \subset \tilde{\mathcal{M}}_{\hookrightarrow}(K) .
$$

This provides an open neighborhood of $Q$ contained in $\tilde{\mathcal{M}}_{\hookrightarrow}(K)$.

\subsection{Countable subbases.}

Proposition 38 (Explicit second-countability). The subsets of the following two forms give a countable subbasis for the topology on $\tilde{\mathcal{M}}$ :

- sets of the form $\tilde{\mathcal{M}}_{\rightsquigarrow}(K)$ where $K \in P C$ is an isomorphism class of a closed rational rectangular union;

- sets of the form $\tilde{\mathcal{M}}_{\hookrightarrow}(U)$ where $U \in P C$ is an isomorphism class of an open rational rectangular union.

Proof. Countability follows from Corollary 33.

The sets listed are clearly open by Theorem 27. We must prove that they form a subbasis for the topology. We will show that the sets in the subbasis used to define the immersive topology in Definition 11 are open in the topology $\mathcal{T}^{\prime}$ generated by the sets listed in this proposition.

Let $P$ be a planar surface and let $K \in \overline{\operatorname{Disk}}(P)$. We will show $\tilde{\mathcal{M}}_{\rightsquigarrow}(K)$ is open in $\mathcal{T}^{\prime}$. Let $Q \in \tilde{\mathcal{M}}_{\rightsquigarrow}(K)$. Then there is an immersion $\iota: K \rightsquigarrow Q$. Let 
$K_{1}=\iota(K)$. Using a closed disk family, we can find a $K_{3} \in \overline{\operatorname{Disk}}(Q)$ so that $K_{1} \subset K_{3}^{\circ}$. Then, Theorem 37 guarantees that there is a closed rational rectangular union $K_{2} \in \overline{\operatorname{Disk}}(Q)$ so that $K_{1} \subset K_{2}^{\circ}$ and $K_{2} \subset K_{3}^{\circ}$. Observe that $Q \in \tilde{\mathcal{M}}_{\rightsquigarrow}\left(K_{2}\right)$ and $\tilde{\mathcal{M}}_{\rightsquigarrow}\left(K_{2}\right) \subset \tilde{\mathcal{M}}_{\rightsquigarrow}(K)$. It follows that $\tilde{\mathcal{M}}_{\rightsquigarrow}(K)$ is open in $\mathcal{T}^{\prime}$.

Let $P$ be a planar surface and let $U \in \overline{\operatorname{Disk}}(P)$. We will show that $\tilde{\mathcal{M}}_{\hookrightarrow \rightarrow}(U)$ is open in $\mathcal{T}^{\prime}$. Let $Q \in \tilde{\mathcal{M}}_{\hookrightarrow \rightarrow}(U)$. Then $U \nrightarrow Q$. Since $U$ is an open disk, we can think of it as a planar surface. Choose a closed disk family $\left\{K_{t}: t>0\right\}$ for $U$. By Proposition 15, there is an $a>0$ so that $K_{a}^{\circ} \nrightarrow Q$. Let $K \in \overline{\operatorname{Disk}}(P)$ be a rectangular union satisfying $K_{a} \subset K^{\circ}$ and $K \subset K_{a+1}^{\circ}$. We have $Q \in \tilde{\mathcal{M}}_{\hookrightarrow \rightarrow}\left(K^{\circ}\right)$ and $\tilde{\mathcal{M}}_{\hookrightarrow \rightarrow}\left(K^{\circ}\right) \subset \tilde{\mathcal{M}}_{\hookrightarrow \rightarrow}(U)$. So, $\tilde{\mathcal{M}}_{\hookrightarrow \rightarrow}(U)$ is open in $\mathcal{T}^{\prime}$.

Proposition 39 (Explicit second-countability of $\tilde{\mathcal{E}}$ ). A countable subbasis for the immersion topology on $\tilde{\mathcal{E}}$ is given by the union of the collection of preimages under $\tilde{\pi}$ of the subbasis provided by Proposition 38 together with the collection sets of the form $\tilde{\mathcal{E}}_{\rightsquigarrow}(K, U)$ where $K \in P C$ is an isomorphism class of a closed rational rectangular union and $U \subset K^{\circ}$ is an open rational rectangle.

Proof. The potential subbasis described is clearly a countable collection of open sets. We must show that it generates the topology. By Proposition 38, the map $\tilde{\pi}$ is continuous in the generated topology. To conclude the proof, we must show that $\tilde{\mathcal{E}}_{\rightsquigarrow}(D, V)$ is open for an arbitrary $D \in \overline{\operatorname{Disk}}(P)$ and arbitrary $U \subset D^{\circ}$ open. Choose a $(Q, q) \in \tilde{\mathcal{E}}_{\rightsquigarrow}(D, V)$. Then there is an immersion $\iota: D \rightsquigarrow Q$ and $q \in \iota(V)$. By taking a closed disk family in $Q$ and applying Theorem 37, we can produce a closed rational rectangular union $K \in \overline{\operatorname{Disk}}(Q)$ so that $\iota(D) \subset K^{\circ}$. Also since $q \in \iota(V)$ and $\iota(V)$ is open, we can find an open rational rectangle $U$ so that $q \in U \subset \iota(V)$. Then, $(Q, q) \in \tilde{\mathcal{E}}_{\rightsquigarrow}(K, U)$. We also claim that $\tilde{\mathcal{E}}_{\rightsquigarrow}(K, U) \subset \tilde{\mathcal{E}}_{\rightsquigarrow}(D, V)$. Suppose $(R, r) \in \tilde{\mathcal{E}}_{\rightsquigarrow}(K, U)$. Then, there is an immersion $j: K \rightsquigarrow R$ and $r \in j(U)$. By composition, we have an immersion $j \circ \iota: D \rightsquigarrow R$. Furthermore, since $U \subset \iota(V)$, we have $r \in j(U) \subset j \circ \iota(V)$.

\section{Sequences}

We have shown that the topologies on $\tilde{\mathcal{M}}$ and $\tilde{\mathcal{E}}$ are second-countable. We recall that a map $f: X \rightarrow Y$ between second-countable spaces is continuous if and only if it is sequentially continuous; see [25, Theorem 30.1]. Therefore, we will begin to use sequences to verify the continuity of maps. We use this section to describe criteria for convergence and consequences of convergence in $\tilde{\mathcal{M}}$ and $\tilde{\mathcal{E}}$.

Proposition 40 (Criterion for convergence in $\tilde{\mathcal{M}}$ ). Let $P \in \tilde{\mathcal{M}}$ be a planar surface and let $\left\langle P_{n}\right\rangle_{n \in \mathbb{N}}$ be a sequence of planar surfaces. Suppose the following two statements hold:

(A) If $D \in \overline{D i s k}(P)$, then $D \rightsquigarrow P_{n}$ for all but finitely many $n$.

(B) If $Q$ is a planar surface, and $Q \hookrightarrow P_{n}$ for infinitely many $n$, then $Q \rightsquigarrow P$. Then, $\left\langle P_{n}\right\rangle$ converges to $P$ in the immersive topology on $\tilde{\mathcal{M}}$.

Example 41. Let $P_{n}$ be the universal cover of $\mathbb{C} \backslash R_{n}$, where $R_{n}$ denotes the set $n$th roots of unity, and where we take the basepoint of $P_{n}$ to be a lift of zero in $\mathbb{C}$. Then $P_{n}$ naturally has a translation structure obtained by pullback under the covering map. We will observe that $P_{n}$ tends to the open unit disk $P$ in the immersive topology. We will check statements of Proposition 40. First of all observe 
that there are embeddings $\epsilon_{n}: P \rightsquigarrow P_{n}$ for all $n$. By restricting this embedding we see any closed disk in $P$ immerses in every $P_{n}$. To see (B) suppose $Q$ is a planar surface which embeds in infinitely many $P_{n}$. Then, $Q$ can be viewed as a subset of some $P_{n}$. It suffices to show that $Q$ is entirely contained in the image $\epsilon_{n}(P)$, since in this case $\epsilon_{n}^{-1}$ restricted to $Q$ is an embedding into $P$. Suppose $Q$ is not contained in $\epsilon_{n}(P)$, we see $Q$ intersects the boundary of $\epsilon_{n}(P)$. Let $\ell$ be the arc length of this interval of intersection viewed as a subset of the unit circle. Choose $M \in \mathbb{N}$ so that for $m>M$, the roots of unity separate the unit circle into intervals of length less than $\ell$. Then for $m>M$ some root of unity lies in the immersed image of $Q$ in the plane. Therefore, $Q \not \leftrightarrow \mathbb{C} \backslash R_{m}$ and thus $Q \not \hookrightarrow P_{m}$ for $m>M$. This contradicts the assumption that $Q$ embeds in infinitely many $P_{m}$.

The following is a direct consequence of Theorem 27. so we will not include a proof.

Corollary 42 (Necessary conditions for convergence in $\tilde{\mathcal{M}})$. Suppose $\left\langle P_{n} \in \tilde{\mathcal{M}}\right\rangle_{n \in \mathbb{N}}$ is a sequence of planar surfaces converging to $P \in \tilde{\mathcal{M}}$. Then, the following two statements are satisfied:

(A') Suppose $K \in P C$ is compact. Then $K \rightsquigarrow P$ implies $K \rightsquigarrow P_{n}$ for $n$ sufficiently large, and $K \hookrightarrow P$ implies $K \hookrightarrow P_{n}$ for $n$ sufficiently large.

(B') Suppose $U \in P C$ is open. Then $U \rightsquigarrow P_{n}$ for infinitely many $n$ implies $U \rightsquigarrow P$, and, $U \hookrightarrow P_{n}$ for infinitely many $n$ implies $U \hookrightarrow P$.

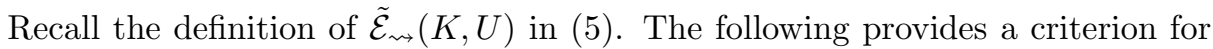
convergence in $\tilde{\mathcal{E}}$.

Proposition 43 (Convergence in $\tilde{\mathcal{E}}$ ). Suppose $P_{n} \in \tilde{\mathcal{M}}$ is a sequence converging to $P \in \tilde{\mathcal{M}}$ in the immersive topology. Let $d_{n}$ denote the Euclidean path metric on $P$. Let $p_{n} \in P_{n}$ and $p \in P$ be a choice of points on these surfaces. Then the following are equivalent:

(1) $\left(P_{n}, p_{n}\right)$ converges to $(P, p)$ in the immersive topology on $\tilde{\mathcal{E}}$.

(2) There is a compact set $K \in P C(P)$ which contains $p$ and an $N$ so that there is an immersion $\iota_{n}: K \rightsquigarrow P_{n}$ defined for $n>N$ so that $d_{n}\left(p_{n}, \iota_{n}(p)\right) \rightarrow 0$ as $n \rightarrow \infty$.

(3) For every compact set $K \in P C(P)$ containing $p$, there is an $N$ and an embedding $e_{n}: K \hookrightarrow P_{n}$ defined for $n>N$ so that $d_{n}\left(p_{n}, e_{n}(p)\right) \rightarrow 0$ as $n \rightarrow \infty$.

Example 44 (Example 41 continued). Let $P_{n}, P$, and $\epsilon_{n}$ be as in Example 41 . Choose $p_{n} \in P_{n}$ for all $n$. We claim that $\left(P_{n}, p_{n}\right)$ converges if there is an $N$ such that $p_{n} \in \epsilon_{n}\left(P_{n}\right)$ for $n>N$ and $\epsilon_{n}^{-1}\left(p_{n}\right)$ converges to some point $p \in P$. In this case $\left(P_{n}, p_{n}\right) \rightarrow(P, p)$. Considering the second statement of Proposition 43, it suffices to choose $K$ to be a closed ball about the origin which contains $p$ in its interior, and consider the second statement for a sequence of values of $U$ where $U$ is an open metric ball about $p$ with radii tending to zero. (Using the definition of the immersive topology, it can be observed that in fact we have given a characterization of convergence of $\left(P_{n}, p_{n}\right)$ to $(P, p)$ for some $p \in P$.)

We note the following consequence.

Corollary 45. Suppose that the sequence $P_{n} \in \tilde{\mathcal{M}}$ converges to $P \in \tilde{\mathcal{M}}$. Then for any $p \in P$, there is an $N$ and a sequence $p_{n} \in P_{n}$ defined for $n>N$ with $\operatorname{Dev}\left(P_{n}, p_{n}\right)=\operatorname{Dev}(P, p)$ so that $\left(P_{n}, p_{n}\right)$ converges to $(P, p)$ in $\tilde{\mathcal{E}}$. 
7.1. Proofs. The following is the proof of our convergence criterion for $\tilde{\mathcal{M}}$.

Proof of Proposition 40, We will suppose statements (A) and (B) of the proposition are satisfied and prove that for any closed disk $K, P \in \tilde{\mathcal{M}}_{\rightsquigarrow}(K)$ implies that $P_{n} \in$ $\tilde{\mathcal{M}}_{\rightsquigarrow}(K)$ for all but finitely many $n$, and that for any open disk $U, P \in \tilde{\mathcal{M}}_{\hookrightarrow}(U)$ implies $P_{n} \in \tilde{\mathcal{M}}_{\hookrightarrow}(U)$ for all but finitely many $n$.

Let $K$ be a closed disk in a planar surface $Q$ and suppose that $P \in \tilde{\mathcal{M}}_{\rightsquigarrow}(K)$. Then there is an immersion $\iota: K \rightsquigarrow P$. By taking a closed disk family in $P$, we can find a closed disk $D$ in that family so that $\iota(K) \subset D$. From $(\mathrm{A})$, we get immersions $\iota_{n}: D \rightsquigarrow P_{n}$ for all but finitely many $n$. Whenever $\iota_{n}$ is defined, the composition $\iota_{n} \circ \iota$ is an immersion $K \rightsquigarrow P_{n}$. This proves $P_{n} \in \tilde{\mathcal{M}}_{\rightsquigarrow}(K)$ for all but finitely many $n$.

Now suppose that $U$ is an open disk in a planar surface $Q$ and that $P \in \tilde{\mathcal{M}}_{\hookrightarrow \rightarrow}(U)$. Then, $U \hookrightarrow P$. Suppose it is not true that $P_{n} \in \tilde{\mathcal{M}}_{\hookrightarrow}(U)$ for all but finitely many $n$. Then there is an increasing sequence of integers $\left\langle n_{k}\right\rangle$ and embeddings $e_{k}: U \hookrightarrow P_{n_{k}}$. Since $U$ is an open disk, it is isomorphic to a planar surface which we abuse notation by also denoting $U$. So, by (B) applied to $U$, we know that there is an immersion $\iota: U \rightsquigarrow P$. Suppose it is not an embedding. Then there are points $u, v \in U$ so that $\iota(u)=\iota(v)$. Let $K \subset U$ be a closed disk in $Q$ containing both $u$ and $v$. Then $\iota(K)$ is compact. As in the prior paragraph, we can find a closed disk $D$ so that $\iota(K) \subset D$. Then, by (A) we get immersions $\iota_{n}: D \rightsquigarrow P_{n}$ for all but finitely many $n$. In particular, for sufficiently large $k$, we have $\left.\iota_{n_{k}} \circ \iota\right|_{K}: K \rightsquigarrow P_{n_{k}}$. But we also get such an immersion as a restriction of an embedding, $\left.e_{k}\right|_{K}: K \hookrightarrow P_{n_{k}}$. Since immersions are unique, it follows that $\left.e_{k}\right|_{K}=\left.\iota_{n_{k}} \circ \iota\right|_{K}$. Therefore, $\left.\iota\right|_{K}$ must be injective, which contradicts the statement above that $\iota(u)=\iota(v)$.

We now prove our equivalences for convergent sequences in $\tilde{\mathcal{E}}$.

Proof of Proposition 43. First we show (1) implies (3). Suppose $\left(P_{n}, p_{n}\right) \rightarrow(P, p)$. Fix a $K \in \mathrm{PC}(P)$ containing $p$. Since $P \rightarrow P$ and $K \hookrightarrow P$, we know that for $n$ sufficiently large there is an embedding $e_{n}: K \hookrightarrow P_{n}$; see Corollary 42, We claim $d_{n}\left(p_{n}, e_{n}(p)\right) \rightarrow 0$. Choose an $\epsilon>0$. Choose a compact disk $D \subset P$ so that $K \subset D$ and so that $p \in D^{\circ}$. Then we can choose an open ball $B$ about $p$ of radius less than $\epsilon$ so that $U \subset D^{\circ}$. Since $(P, p) \in \tilde{\mathcal{E}}_{\rightsquigarrow}(D, U)$, we know that there is an $N$ so that $\left(P_{n}, p_{n}\right) \in \tilde{\mathcal{E}}_{\rightsquigarrow}(D, U)$ for $n>N$. But then there is an immersion $\iota_{n}: D \rightsquigarrow P_{n}$ so that $p_{n} \in \iota_{n}(U)$. But since $U$ is an small ball about $p$, we know that this implies that $d_{n}\left(p_{n}, \iota_{n}(p)\right)<\epsilon$. Finally, since immersions are unique, we know $\iota_{n}=e_{n}$ on $K$, so the same holds with $e_{n}(p)$ replacing $\iota_{n}(p)$.

Clearly (3) implies (2). We will finish the proof by showing that (2) implies (1). Let $K, N$ and $\iota_{n}$ be as in (2). By definition of the immersive topology, we need to show that for every closed disk $D \in \mathrm{PC}$ and every open $U \subset D^{\circ}$ so that $(P, p) \in \tilde{\mathcal{E}}_{\rightsquigarrow}(D, U)$, we have $\left(P_{n}, p_{n}\right) \in \tilde{\mathcal{E}}_{\rightsquigarrow}(D, U)$ for $n$ sufficiently large. Fix $D$ and $U$ and suppose $(P, p) \in \tilde{\mathcal{E}}_{\rightsquigarrow}(D, U)$ so that there is an immersion $j: D \rightsquigarrow P$ and $p \in j(U)$. Since $j(U)$ is open, there is an $\epsilon>0$ so that the open $\epsilon$ ball about $p$ is contained in $j(U)$. Choose a closed disk $K^{\prime} \in P$ so large that it contains both $K$ and $j(D)$. Then since $P_{n} \rightarrow P$, we know that there is an $N^{\prime}>N$ and immersions $\iota_{n}^{\prime}: K^{\prime} \rightarrow P_{n}$ for $n>N^{\prime}$. Observe that the image $\iota_{n}^{\prime} \circ j(U)$ contains an $\epsilon$-ball about $\iota_{n}^{\prime}(p)$ because $\iota_{n}^{\prime}$ is a local translation and $j(U)$ contains such a ball about $p$. By uniqueness of immersions we know $\left.\iota_{n}^{\prime}\right|_{K}=\iota_{n}$, and in particular $\iota_{n}(p)=\iota_{n}^{\prime}(p)$. 
By hypothesis, there is an $N^{\prime \prime}>N^{\prime}$ so that for $n>N^{\prime \prime}$ we have $d_{n}\left(p_{n}, \iota_{n}(p)\right)<\epsilon$ which then implies $p_{n} \in \iota_{n}^{\prime} \circ j(U)$ by prior remarks. Observe that when $n>N^{\prime \prime}$, we have that $\left.\iota_{n}^{\prime} \circ j\right|_{D}$ is an immersion of $D$ into $P_{n}$ and $p_{n} \in \iota_{n}^{\prime} \circ j(U)$ so that $n>N^{\prime \prime}$ implies $\left(P_{n}, p_{n}\right) \in \tilde{\mathcal{E}}_{\rightsquigarrow}(D, U)$ as desired.

Proof of Corollary 45. Let $K \subset P$ be a closed disk containing $p$ in its interior. Let $\iota_{n}: K \rightarrow P_{n}$ be immersions guaranteed to exist for $n$ sufficiently large because $P_{n} \rightarrow P$ and $\tilde{\mathcal{M}}_{\rightsquigarrow}(K)$ is open. Let $p_{n}=\iota_{n}(p)$. Then we see by definition that $\left(P_{n}, p_{n}\right) \in \tilde{\mathcal{E}}_{\rightsquigarrow}(K, U)$ whenever $\iota_{n}$ is defined and when $p$ lies in the open subset $U \subset K^{\circ}$. The developing map comment is true because Dev is invariant under immersions.

\section{Continuity of immersions}

The following explains that immersions and embeddings are jointly continuous in choice of the domain and range, and that the natural domains for these maps are closed.

Theorem 46. The sets

$$
\{(P, Q) \in \tilde{\mathcal{M}} \times \tilde{\mathcal{M}}: P \rightsquigarrow Q\} \quad \text { and } \quad\{(P, Q) \in \tilde{\mathcal{M}} \times \tilde{\mathcal{M}}: P \hookrightarrow Q\}
$$

are closed in $\tilde{\mathcal{M}} \times \tilde{\mathcal{M}}$. The function

$$
I:\{((P, p), Q) \in \tilde{\mathcal{E}} \times \tilde{\mathcal{M}}: P \rightsquigarrow Q\} \rightarrow \tilde{\mathcal{E}}
$$

which sends $((P, p), Q)$ to the image of $p$ under the immersion $P \rightsquigarrow Q$ has a closed domain and is continuous.

Proof of Theorem 46. We begin with dealing with the first sentence in the case of embeddings. Suppose $\left\{P_{n}\right\}$ and $\left\{Q_{n}\right\}$ are sequences of planar surfaces each of which converges to $P$ and $Q$, respectively, and $P_{n} \hookrightarrow Q_{n}$ for all $n$. We need to show $P \hookrightarrow Q$. It suffices to show that any closed disk $D$ in $P$ embeds in $Q$ by Proposition 15. Let $K \subset P$ be a closed disk containing $D$ in its interior. Observe that $K$ embeds in all but finitely many $P_{n}$ since $\tilde{\mathcal{M}}_{\hookrightarrow}(K)$ is open. Then by composition with $P_{n} \hookrightarrow Q_{n}, K \hookrightarrow Q_{n}$ for infinitely many $n$. Since $\tilde{\mathcal{M}}_{\hookrightarrow \rightarrow}\left(K^{\circ}\right)$ is open, we see that $K^{\circ}$ embeds in $Q$, and thus so does $D$.

For the case of immersions we need rectangular unions. Suppose $P_{n} \rightarrow P$, $Q_{n} \rightarrow Q$ and each $P_{n} \rightsquigarrow Q_{n}$. We will show $P \rightsquigarrow Q$. Again it suffices to show that every open disk $D$ with compact closure in $P$ immerses in $Q$. Let $D \subset P$ be such a disk, and let $V \subset P$ be an open rectangular union containing $D$ which is a topological disk with compact closure $\bar{V}$. Since $\tilde{\mathcal{M}}_{\hookrightarrow}(\bar{V})$ is open by Theorem 27. there are embeddings $e_{n}: \bar{V} \hookrightarrow P_{n}$ for $n$ sufficiently large. By composing with immersions $P_{n} \rightsquigarrow Q_{n}$, we see $V \rightsquigarrow Q_{n}$ for all but finitely many $n$. By Corollary 32. there are only finitely many such images of $V$ up to isomorphism. Let $W$ be an isomorphism class which appears infinitely many times. By Theorem 27, the set $\tilde{\mathcal{M}}_{\hookrightarrow}(W)$ is open, so it must be that $W \hookrightarrow Q$. Note that since $V \rightsquigarrow W$ we have $D \rightsquigarrow W$. Thus $D \rightsquigarrow Q$, which completes the proof that $P \rightsquigarrow Q$.

We note that the domain of $I$ is the preimage of $\{(P, Q): P \rightsquigarrow Q\}$ under $\tilde{\pi} \times$ id where $\tilde{\pi}: \tilde{\mathcal{E}} \rightarrow \tilde{\mathcal{M}}$ is the projection. Thus the domain of $I$ is closed.

Now let $\left(\left(P_{n}, p_{n}\right), Q_{n}\right)$ be a sequence in the domain of $I$ which converges to $((P, p), Q) \in \tilde{\mathcal{E}} \times \tilde{\mathcal{M}}$. So there are immersions $\iota_{n}: P_{n} \rightsquigarrow Q_{n}$, and there is an 
immersion $\iota: P \rightsquigarrow Q$ by remarks above. We need to show that $\left(Q_{n}, \iota_{n}\left(p_{n}\right)\right) \rightarrow$ $(Q, \iota(p))$. Choose $K \subset P$ compact and containing $p$ in its interior. It follows that there is an $\epsilon>0$ so that $K^{\circ}$ contains an $\epsilon$-ball about $p$. By Proposition 43 we know that for $n$ sufficiently large there is an embedding $e_{n}: K \hookrightarrow P_{n}$ and $d_{P_{n}}\left(p_{n}, e_{n}(p)\right) \rightarrow 0$ as $n \rightarrow \infty$. When this distance is less than $\epsilon$, we know that

$$
d_{P_{n}}\left(e_{n}^{-1}\left(p_{n}\right), p\right)=d_{P_{n}}\left(p_{n}, e_{n}(p)\right),
$$

so the quantity at left also tends to zero. Let $K^{\prime}=\iota(K)$ which is compact. Since we know $Q_{n} \rightarrow Q$, for $n$ sufficiently large there is an embedding $f_{n}: \iota(K) \hookrightarrow Q_{n}$. Observe that $f_{n} \circ \iota: K \rightsquigarrow Q_{n}$. Since immersions non-strictly contract distances, by applying this immersion we see

$$
d_{Q_{n}}\left(f_{n} \circ \iota \circ e_{n}^{-1}\left(p_{n}\right), f_{n} \circ \iota(p)\right) \rightarrow 0 \text { as } n \rightarrow \infty .
$$

Now observe that both $f_{n} \circ \iota \circ e_{n}^{-1}$ and $\left.\iota_{n}\right|_{\iota(K)}$ give immersions of $\iota(K) \rightsquigarrow Q_{n}$ so they are equal. It therefore follows that $d_{Q_{n}}\left(\iota_{n}\left(p_{n}\right), f_{n} \circ \iota(p)\right) \rightarrow 0$ as $n \rightarrow \infty$. Then by Proposition 43 it follows that $\left(Q_{n}, \iota_{n}\left(p_{n}\right)\right) \rightarrow(Q, \iota(p))$ as desired.

\section{Compact SubSets AND Metrizability}

In this section, we prove the following theorem and establish consequences such as metrizability of $\tilde{\mathcal{M}}$ and $\tilde{\mathcal{E}}$.

Theorem 47. Let $P$ be a planar surface. The set of surfaces

$$
\tilde{\mathcal{M}} \backslash \tilde{\mathcal{M}}_{\nsim}(P)=\{Q \in \tilde{\mathcal{M}}: P \rightsquigarrow Q\}
$$

is compact.

It follows the only way a sequence $\left\langle P_{n}\right\rangle$ of planar surfaces can leave every compact set of $\tilde{\mathcal{M}}$ is if the radius of largest open Euclidean metric ball we can immerse in $P_{n}$ centered at the basepoint tends to zero as $n \rightarrow \infty$.

We establish two important consequences of this result.

Corollary 48 (Local compactness). Both $\tilde{\mathcal{M}}$ and $\tilde{\mathcal{E}}$ are locally compact in the sense that every point in these spaces has a compact neighborhood.

Corollary 49 (Metrizability). The spaces $\tilde{\mathcal{M}}$ and $\tilde{\mathcal{E}}$ are metrizable.

9.1. Direct limits. For the proof of Theorem 47, we need to know that an $\rightsquigarrow-$ increasing sequence converges.

Proposition 50 (Direct limit). Suppose $\left\langle P_{n} \in \tilde{\mathcal{M}}\right\rangle_{n \geq 1}$ is a sequence satisfying

$$
P_{1} \rightsquigarrow P_{2} \rightsquigarrow P_{3} \rightsquigarrow \ldots
$$

Then, the sequence converges to $\bigvee\left\{P_{n}\right\}$.

Proof. Let $\left\langle P_{n}\right\rangle_{n \geq 1}$ be a sequence of planar surfaces as stated in the proposition. Then for each $m, n$ with $m \leq n$, there is an immersion $\iota_{m, n}: P_{m} \rightsquigarrow P_{n}$. Let $P_{\infty}=\Upsilon\left\{P_{n}\right\}$. By the Fusion Theorem, there are immersions $j_{n}: P_{n} \rightarrow P_{\infty}$. To prove that the sequence $\left\langle P_{n}\right\rangle$ converges to $P_{\infty}$, we apply the convergence criterion of Proposition 40.

Let $K \in \overline{\operatorname{Disk}}\left(P_{\infty}\right)$. The set $K$ is compact, and we will apply a compactness argument to say that there is an $M$ and a lift $\tilde{K} \subset P_{M}$ containing the basepoint of $P_{M}$ so that $\left.j_{M}\right|_{\tilde{K}}$ is a homeomorphism from $\tilde{K}$ onto $K$ which respects the basepoints. Then, the inverse of this restriction $\left(\left.j_{M}\right|_{\tilde{K}}\right)^{-1}$ is the needed immersion of 
$K$ into $P_{M}$. We can then immerse $K$ into all $P_{N}$ with $N>M$ by composing with $\iota_{M, N}$. This will prove the first statement needed from Proposition 40 .

First we claim that $P_{\infty}=\bigcup_{n} j_{n}\left(P_{n}\right)$. To see this, let $Q=\bigcup_{n} j_{n}\left(P_{n}\right)$ which is a path connected open subset of $P_{\infty}$ containing the basepoint. Then the universal cover $\tilde{Q}$ is a planar surface. Observe that $j_{n}$ lifts to an immersion $\tilde{j}_{n}: P_{n} \rightarrow \tilde{Q}$ for each $n$. But then by statement (II) of Theorem 21 we have $P_{\infty} \rightsquigarrow \tilde{Q}$ since $P_{\infty}=\curlyvee\left\{P_{n}\right\}$. The universal covering map $\tilde{Q} \rightarrow Q \subset P_{\infty}$ gives an immersion $\tilde{Q} \rightsquigarrow P_{\infty}$. Since $\tilde{Q} \rightsquigarrow P_{\infty}$ and $P_{\infty} \rightsquigarrow \tilde{Q}$ we see $\tilde{Q}=P_{\infty}$ as planar surfaces, and that the immersion $\tilde{Q} \rightarrow P_{\infty}$ coming from the covering map of $Q$ actually an isomorphism of planar surfaces. Since $Q$ is the image of this immersion, we see $Q=P_{\infty}$ as claimed.

We will now construct $\tilde{K}$. As $P_{\infty}=\bigcup_{n} j_{n}\left(P_{n}\right)$, for each $x \in K$, there is an $n(x) \geq 1$ and a $p(x) \in P_{n(x)}$ so that $j_{n(x)}(p(x))=x$. For the basepoint of $P_{\infty}$ in $K$, we take $n(x)=1$ and $p(x)$ to be the basepoint of $P_{1}$. For each $x$, let $B_{x} \subset P_{n(x)}$ be an open metric ball about $p(x)$ so that $\left.D e v\right|_{B_{x}}$ is a homeomorphism onto a ball in the plane with the same radius. Then the collection of images $\left\{j_{n(x)}\left(B_{x}\right): x \in K\right\}$ is an open cover of $K$. So there is a finite subcover indexed by the subset $\left\{x_{1}, \ldots, x_{k}\right\} \subset K$. We add the basepoint of $P_{\infty}$ to this set and call it $x_{0}$. Consider the collection

$$
\mathcal{I}=\left\{(i, j) \in\{0, \ldots, k\}^{2}: j_{n\left(x_{i}\right)}\left(B_{x_{i}}\right) \cap j_{n\left(x_{j}\right)}\left(B_{x_{j}}\right) \neq \emptyset\right\} .
$$

Then for each $(i, j) \in \mathcal{I}$, we can choose points $y \in B_{x_{i}}$ and $z \in B_{x_{j}}$ so that $j_{n\left(x_{i}\right)}(y)=j_{n\left(x_{j}\right)}(z)$. By Corollary 24, there is a finite subset $\mathcal{F} \subset\left\{P_{n}\right\}$ containing $P_{n\left(x_{i}\right)}$ and $P_{n\left(x_{j}\right)}$ so that the immersions $P_{n\left(x_{i}\right)} \rightsquigarrow \curlyvee \mathcal{F}$ and $P_{n\left(x_{j}\right)} \rightsquigarrow \curlyvee \mathcal{F}$ send $y$ and $z$ to the same point. Because we are working with a directed sequence, we just have $\bigvee \mathcal{F}=P_{N(i, j)}$ where $N(i, j)$ is the maximal index of a planar surface in $\mathcal{F}$. So, there is an $N=N(i, j)$ so that $\iota_{m\left(x_{i}\right), N}(y)=\iota_{m\left(x_{j}\right), N}(z)$. Then, because $j_{n\left(x_{i}\right)}\left(B_{x_{i}}\right) \cap j_{n\left(x_{j}\right)}\left(B_{x_{j}}\right)$ is path connected, the map $j_{N(i, j)}$ restricted to

$$
\iota_{n\left(x_{i}\right), N(i, j)}\left(B_{x_{i}}\right) \cup \iota_{n\left(x_{j}\right), N(i, j)}\left(B_{x_{j}}\right)
$$

is injective. We have defined $N(i, j)$ for all $(i, j) \in \mathcal{I}$. Let $M=\max _{(i, j) \in \mathcal{I}} N(i, j)$. Then $j_{M}$ restricted to

$$
\bigcup_{i=0}^{k} \iota_{n\left(x_{i}\right), M}\left(B_{x_{i}}\right)
$$

is injective. Then, because $j_{M}$ is a local homeomorphism, this restriction is a homeomorphism onto its image. The image contains $K$. We conclude that we can set $\tilde{K}$ equal to the preimage of $K$ under this restriction of $j_{M}$. This verifies the existence of $\tilde{K}$ and proves that the first statement of Proposition 40 holds.

Now we consider the second statement of Proposition 40. Suppose that $Q$ is a planar surface, and there is an immersion $k: Q \rightsquigarrow P_{n}$ for some $n$. Then, $j_{n} \circ k: Q \rightsquigarrow$ $P_{\infty}$. This proves the second statement needed from Proposition 40, and concludes the proof that $P_{n} \rightarrow P_{\infty}$.

\subsection{Proofs.}

Proof of Theorem 47, Let $P$ be a planar surface. We will prove $\tilde{\mathcal{M}} \backslash \tilde{\mathcal{M}}_{\varkappa \rightarrow}(P)$ is compact. Since $\tilde{\mathcal{M}}$ is second-countable, so is the subspace $\tilde{\mathcal{M}} \backslash \tilde{\mathcal{M}}_{\varkappa}(P)$. In the presence of second-countability, compactness is implied by sequentially compactness, i.e., that every sequence in $\tilde{\mathcal{M}} \backslash \tilde{\mathcal{M}}_{\varkappa}(P)$ has a convergent subsequence 
[20, Chapter 11, Theorem 1.10]. So, let $\left\langle Q_{n}\right\rangle_{n \geq 0}$ be a sequence in $\tilde{\mathcal{M}} \backslash \tilde{\mathcal{M}}_{\varkappa \varkappa}(P)$. We will provide an algorithm which produces a convergent subsequence $\left\langle Q_{n_{k}}\right\rangle_{k \geq 0}$ converging to some limit $R \in \tilde{\mathcal{M}} \backslash \tilde{\mathcal{M}}_{\varkappa}(P)$.

Recall that there are only countably many open rational rectangular unions which are homeomorphic to open disks. See Corollary 33 . Let $\left\langle P_{m} \in \tilde{\mathcal{M}}\right\rangle_{m \geq 1}$ be a sequence which enumerates all of these rectangular unions. We will construct a subsequence $\left\langle P_{m_{k}}\right\rangle$ of $\left\langle P_{m}\right\rangle$ while simultaneously producing $\left\langle Q_{n_{k}}\right\rangle$.

Our algorithm is really an inductive sequence of the following definitions.

(1) Set $R_{0}=P$.

(2) Set $m_{0}, n_{0}=0$ and $k=1$.

(3) Set $\mathcal{I}_{0}=\{n: n \geq 1\}$.

(4) For each successive integer $m \geq 1$, if $P_{m} \rightsquigarrow Q_{n}$ for infinitely many $n \in \mathcal{I}_{k}$, then perform the following steps:

(a) Set $m_{k}=m$.

(b) Set $R_{k}=R_{k-1} \curlyvee P_{m_{k}}$.

(c) Set $n_{k}=\min \left\{n \in \mathcal{I}_{k-1}: P_{m_{k}} \rightsquigarrow Q_{n}\right\}$.

(d) Set $\mathcal{I}_{k}=\left\{n \in \mathcal{I}_{k-1}: P_{m_{k}} \rightsquigarrow Q_{n}\right.$ and $\left.n>n_{k}\right\}$.

(e) Increment $k$. (Reassign $k$ to be $k+1$.)

Observe that by definition of the fusion, $R_{k-1} \rightsquigarrow R_{k}$ for all $k \geq 1$. So by taking a direct limit, we can define $R=\lim _{k \rightarrow \infty} R_{k}$; see Proposition 50. We make several further remarks about this construction:

(R1) For each $k$ and each $l \geq k, P_{m_{k}} \rightsquigarrow Q_{n_{l}}$. (Proof: This holds when $k=l$ by definition of $m_{k}$ and $n_{k}$. It holds when $l>k$, because each such $n_{l}$ lies in $\left.\mathcal{I}_{k} \cdot\right)$

(R2) We have $R_{k}=P \curlyvee P_{m_{1}} \curlyvee \ldots \curlyvee P_{m_{k}}$.

(R3) For each $k$ and each $l \geq k, R_{k} \rightsquigarrow Q_{n_{l}}$. (Proof: By (R1), each $P_{m_{j}} \rightsquigarrow Q_{n_{l}}$ for $j \leq k$. So by the Fusion Theorem and (R2), $R_{k} \rightsquigarrow Q_{n_{l}}$.)

We claim that the subsequence $\left\langle Q_{n_{l}}\right\rangle$ also converges to $R$. To prove this, we will use the convergence criterion of Proposition 40. First suppose that $K \in \overline{\operatorname{Disk}}(R)$. We will prove that $K \rightsquigarrow Q_{n_{l}}$ for $l$ sufficiently large. Since $\left\langle R_{k}\right\rangle$ converges to $R$, there is an $L$ so that $K \rightsquigarrow R_{l}$ for $l>L$. So by composing these immersions with the immersions given by remark (R3), we see $K \rightsquigarrow Q_{n_{l}}$ for $l>L$.

Now let $U$ be a planar surface. We will show that if $U$ immerses in infinitely many $Q_{n_{k}}$, then $U \rightsquigarrow R$. By Proposition 15, it suffices to prove that every compact disk $K \in \overline{\operatorname{Disk}}(U)$ immerses in $R$. Fix $K \in \overline{\operatorname{Disk}}(U)$. By Theorem 37, there is an open rational rectangular union in $\operatorname{Disk}(U)$ which contains $K$. By definition of $P_{m}$, there is an $m$ so that $P_{m}$ is isomorphic to this union. In particular $P_{m} \rightsquigarrow U$. Since $U \rightsquigarrow Q_{n_{k}}$ for infinitely many $k$, it must be true that $P_{m}$ immerses in infinitely many $Q_{n_{k}}$. It follows that $P_{m}=P_{m_{k}}$ for some $k$. Then, by the definition of the fusion, $P_{m_{k}} \rightsquigarrow R_{k}$. Because $R_{k} \rightsquigarrow R$, we know $P_{m_{k}} \rightsquigarrow R$. Finally, because $K \rightsquigarrow P_{m_{k}}$, we know that $K \rightsquigarrow R$.

Proof of Corollary 48 . To see $\tilde{\mathcal{M}}$ is locally compact, choose $P \in \tilde{\mathcal{M}}$. Let $K$ be a closed disk in $P$. Then

$$
U=\left\{Q \in \tilde{\mathcal{M}}: K^{\circ} \rightsquigarrow Q\right\}
$$

is a compact and contains the neighborhood $\tilde{\mathcal{M}}_{\rightsquigarrow}(K)$ of $P$.

Since $\tilde{\mathcal{M}}$ is locally compact, so is $\tilde{\mathcal{M}} \times \mathbb{R}^{2}$. We will show that the map $\tilde{\mathcal{E}} \rightarrow \tilde{\mathcal{M}} \times \mathbb{R}^{2}$ defined by $(P, p) \mapsto(P, \operatorname{Dev}(p))$ is a local homeomorphism. Local compactness 
of $\tilde{\mathcal{E}}$ follows because we can pullback compact neighborhoods. The map is clearly continuous since both $\tilde{\pi}$ and Dev are continuous. It is locally continuously invertible because any $(P, p)$ lies in a set of the form $\tilde{\mathcal{E}}_{\rightsquigarrow}(K, U)$ where $U$ is so small that $\left.D e v\right|_{U}$ is a homeomorphism onto its image. So, we can recover a point $(Q, q) \in \tilde{\mathcal{E}}_{\rightsquigarrow}(K, U)$ from its image $(Q, \operatorname{Dev}(q))$ by selecting $u \in U$ to be the unique point so that $\operatorname{Dev}(u)=\operatorname{Dev}(q)$. Then there is an immersion $\iota: K \rightsquigarrow Q$ and $\iota(u)=q$. Continuity of this local inverse is then provided by Theorem 46 .

Proof of Corollary 49 . Since $\tilde{\mathcal{M}}$ and $\tilde{\mathcal{E}}$ are locally compact and Hausdorff, they are regular [25, Exercise 32.3]. Since they are second-countable and regular, they are metrizable by Urysohn's Metrization Theorem [25, Theorem 34.1].

\section{Appendix A. Comparison to McMullen's geometric topology}

McMullen has established a geometric topology on the space of all Riemann surfaces equipped with a base-frame paired with a holomorphic quadratic differential where the Riemann surface is allowed to be of arbitrary topological type [24, Appendix]. In brief, McMullen first provides a geometric topology on the space of all Riemann surfaces with a base-frame (a choice of a tangent vector at some basepoint) by identifying such surfaces with a quotient of a simply connected rotationally symmetric domain (disk, plane, or sphere) in the Riemann sphere by a subgroup of $P S L(2, \mathbb{C})$. This identification is done via the unique uniformization carrying a lift $v$ to the universal cover to the tangent vector 1 at the origin. Then given a triple $(X, v, q)$ where $(X, v)$ is a Riemann surface with a base-frame and $q$ is a holomorphic quadratic differential, McMullen considers the lift of $q$ to a holomorphic quadratic differential $\tilde{q}$ on the universal cover as identified with the domain mentioned above. Such differentials then correspond to the choice of a holomorphic function on the domain satisfying $\tilde{q}=\phi(z) d z^{2}$. The space of pairs $(X, q)$ is then topologized in such a way so that sequences converge if the underlying Riemann surfaces converge and the functions $\phi$ constructed as above converge uniformly on compact sets. See [24, Appendix] for a detailed description of the geometric topology.

McMullen's topology differs from the topology here in a philosophical way. The immersive topology introduced here is intended to be a "geometric topology for the flat structure" and was designed to impose the minimal amount of structure necessary for making certain elementary geometric arguments (through the use of immersions and embeddings). In contrast, McMullen's geometric topology makes convergence of underlying Riemann surfaces necessary for convergence of flat structures.

We will demonstrate in this section that our approach gives a less strict notion of convergence. However, it will follow from work in subsequent papers on the immersive topology that this topology agrees with McMullen's geometric topology on natural subspaces such as on the closures of strata of finite genus translation surfaces.

Observe there are differences in the spaces being topologized by the geometric topology and the immersive topology. The geometric topology works with quadratic differentials with zeros, while our topology only applies to surfaces with a translation structure and no singularities. So, we hope to compare the immersive topology on the space of all translation surfaces with the geometric topology applied to an appropriate subset. Unfortunately, our planar surfaces involve only the 
choice of a basepoint, so there is freedom in the choice of a triple $(X, v, q)$ representing a planar surface $P$ corresponding to the choice of a non-zero vector in the tangent plane at the basepoint. This is a minor point, but must be dealt with to make a precise statement relating the topologies. We show the following.

Theorem 51. There is a sequence of planar surfaces $P_{n}$ which is convergent in the immersive topology so that for any sequence $\left(X_{n}, v_{n}, q_{n}\right)$ translation equivalent to $P_{n}$, no subsequence of $\left(X_{n}, v_{n}, q_{n}\right)$ converges to a representation of a flat surface in the geometric topology.

Remark 52. As a consequence of this theorem, in the geometric topology the space of flat surfaces with a lower bound on the injectivity radius is not compact. This property holds for the immersive topology by Theorem 47

Our construction utilizes the following elementary observation in complex analysis.

Proposition 53. Suppose $P$ is a planar surface uniformized by the plane, and $p \in P$ is a point distinct from the basepoint. Let $\tilde{P}$ be the universal cover of $P \backslash p$. Then $\tilde{P}$ is also uniformized by the plane.

Essentially the uniformizing map of $\tilde{P}$ can be expressed as the uniformizing map for $P$ precomposed with the exponential map. We will not provide a formal proof.

Proof of Theorem 51. First we produce the sequence $P_{n}$ inductively. Choose a countable dense subset $\left\{z_{i}: i=0,1,2, \ldots\right\}$ of the unit circle. For each $n \geq 0$, let $D_{n}$ be the closed unit disk with $\left\{z_{i}: 0 \leq i<n\right\}$ removed. Define $D_{-1}$ to be the closed unit disk. Let $P_{0}$ be the plane viewed as a planar surface. Observe that there is an embedding of the closed unit disk, $\epsilon_{0}: D_{-1} \hookrightarrow P_{0}$. Now we will begin our induction. Assume that $P_{n}$ is uniformized by the plane, and there is an embedding $\epsilon_{n}: D_{n-1} \hookrightarrow P_{n}$. Define $p_{n}=\epsilon_{n}\left(z_{n}\right)$. Then let $P_{n+1}$ be the universal cover of $P_{n} \backslash\left\{p_{n}\right\}$. By the proposition above, $P_{n+1}$ is also uniformized by the plane. By restriction we see that $D_{n} \hookrightarrow P_{n}$. Since $D_{n}$ is simply connected this embedding lifts to an embedding $\epsilon_{n+1}: D_{n} \hookrightarrow P_{n+1}$. This defines the sequence $P_{n}$.

We will now verify convergence in the immersive topology. Let $E_{i}$ be the union of the open unit disk and the singleton $\left\{z_{i}\right\}$. Observe that by construction $E_{i} \triangleleft P_{i}$. In fact, $E_{i} \leftrightarrow P_{n}$ for $n \geq i$. This may be seen by induction; each $P_{n+1}$ immerses in $P_{n}$ by construction since $P_{n+1}$ is a covering of $P_{n}$ with a point removed. So, if there was an embedding of $E_{i}$ into $P_{n}$ for $n \geq i$, then composing with a finite list of immersions would give an immersion of $E_{i}$ into $P_{i}$, but such an immersion must be an embedding which is a contradiction. It then follows that as planar surfaces $P_{n}$ converges to the unit disk in the immersive topology, since no open disk larger than the open unit disk can be immersed in infinitely many $P_{n}$; compare Example 41

Now we will consider the behavior of the sequence in the geometric topology. Select for each $P_{n}$ a uniformizing map $h_{n}: \mathbb{C} \rightarrow P_{n}$ carrying 0 to the basepoint of $P_{n}$. (This involves a choice for each $n$.) Let $f_{n}: \mathbb{C} \rightarrow \mathbb{C}$ be Dev $\circ h_{n}$. Let $q_{n}=f_{n}^{\prime}(z)^{2} d z^{2}$. Suppose after passing to a subsequence, $\left(\mathbb{C}, v, q_{n_{k}}\right)$ converges (where $v$ is the tangent vector 1 based at zero). The limit is then of the form $(\mathbb{C}, v, q)$ for some quadratic differential $q$ on $\mathbb{C}$ and we know by definition of the topology that $q=\phi(z) d z^{2}$ and $f_{n_{k}}^{\prime}(z)^{2}$ converges to $\phi$ uniformly on compact sets. By passing to another subsequence (because of the square), we can assume that 
$f_{n_{k}}(z)$ converges to a holomorphic function $f: \mathbb{C} \rightarrow \mathbb{C}$ satisfying $f(0)=0$ uniformly on compact sets.

Now assume that the limiting differential represents a surface. Then, in particular, $f$ is not identically zero, and so $f$ misses at most one point of the plane. In particular, there is a $z \in \mathbb{C}$ so that $|f(z)|>1$. Then $\left|f_{n_{k}}(z)\right|>1$ for $k$ sufficiently large. Consider the embedding $\epsilon_{n_{k}}: D_{n_{k}-1} \hookrightarrow P_{n}$ and recall that $\epsilon_{n_{k}}$ cannot be extended to a larger subset of the closed disk. Let $\Gamma_{k}$ be the intersection of $D_{n_{k}-1}$ with the unit circle, which consists of $n_{k}-1$ arcs of the unit circle running between the points $\left\{z_{i}: 0 \leq i<n_{k}-1\right\}$. Let $C_{k}=f_{n_{k}}^{-1} \circ \epsilon_{n_{k}}\left(\Gamma_{k}\right)$, which consists of $n_{k}-1$ disjoint arcs in $\mathbb{C}$ which extend to closed arcs in the Riemann sphere $\hat{\mathbb{C}}$ initiating and terminating at $\infty$. For $k$ sufficiently large, one of these arcs, say $c_{k} \subset C_{k}$, separates 0 from $z$ since $\left|f_{n_{k}}(z)\right|>1$. Choose a large closed ball about the origin $B \subset \mathbb{C}$ so that $z$ lies in the interior of $B$. Then by passing to a subsequence, we can assume that the intersections $c_{k} \cap B$ converge in the Hausdorff topology on closed subsets of $B$ to some closed subset $c \subset B$. Each of $c_{k} \cap B$ has some definite length since $c_{k}$ separates 0 from $z$. The length of the associated arc $\gamma_{k} \subset \Gamma_{k}$ of $D_{n_{k}-1}$ can be computed in terms of $f_{n_{k}}^{\prime}$ as

$$
\int_{c_{k}}\left|f_{n_{k}}^{\prime}(z)\right| d z
$$

Because of our choice of points $\left\{z_{i}\right\}$ dense in the circle, the length of $\gamma_{k}$ tends to zero, so these integrals tend to zero. Let $w \in c \subset B$. If $|f(w)|>0$, then there would be an $\epsilon>0$ and a definite neighborhood $W$ about $w$ so that $\left|f_{n_{k}}\left(w^{\prime}\right)\right|>\epsilon$ for $w^{\prime} \in W$ and $k$ large enough, but this contradicts the convergence of (9) to zero. Therefore, $f$ is identically zero on $c$, but this violates the fact that non-zero holomorphic functions have isolated zeros.

\section{ACKNOWLEDGMENTS}

The author would like to thank Joshua Bowman for helpful conversations at the beginning of this work, Anja Randecker for numerous helpful comments, and the anonymous referee for making numerous helpful suggestions. The author thanks Matt Bainbridge for pointing out McMullen's geometric topology. The impetus for writing this article came from the conference "International conference and workshop on surfaces of infinite type" held at Centro de Ciencias Matemáticas de la UNAM in Morelia, Mexico. Early versions of this paper were written while visiting the Institute for Computational and Experimental Research in Mathematics (ICERM).

\section{REFERENCES}

[1] Garrett Birkhoff, Lattice theory, Third edition. American Mathematical Society Colloquium Publications, Vol. XXV, American Mathematical Society, Providence, R.I., 1967. MR0227053

[2] Joshua P. Bowman and Ferrán Valdez, Wild singularities of flat surfaces, Israel J. Math. 197 (2013), no. 1, 69-97, DOI 10.1007/s11856-013-0022-y. MR3096607

[3] R. Chamanara, Affine automorphism groups of surfaces of infinite type, In the tradition of Ahlfors and Bers, III, Contemp. Math., vol. 355, Amer. Math. Soc., Providence, RI, 2004, pp. 123-145, DOI 10.1090/conm/355/06449. MR2145060

[4] Lucien Clavier, Anja Randecker, and Chenxi Wu, Rotational component spaces for infinitetype translation surfaces, arXiv preprint arXiv:1412.0633 (2014). 
[5] Vincent Delecroix, Pascal Hubert, and Samuel Lelièvre, Diffusion for the periodic wind-tree model (English, with English and French summaries), Ann. Sci. Éc. Norm. Supér. (4) 47 (2014), no. 6, 1085-1110, DOI 10.24033/asens.2234. MR.3297155

[6] Ralph H. Fox and Richard B. Kershner, Concerning the transitive properties of geodesics on a rational polyhedron, Duke Math. J. 2 (1936), no. 1, 147-150, DOI 10.1215/S0012-7094-3600213-2. MR 1545913

[7] Krzysztof Frączek and Corinna Ulcigrai, Non-ergodic $\mathbb{Z}$-periodic billiards and infinite translation surfaces, Invent. Math. 197 (2014), no. 2, 241-298, DOI 10.1007/s00222-013-0482-z. MR 3232007

[8] George Grätzer, Lattice theory: foundation, Birkhäuser/Springer Basel AG, Basel, 2011. MR2768581

[9] W. Patrick Hooper, Immersions and the space of all translation structures, 2013, arXiv:1310.5193.

[10] W. Patrick Hooper, Immersions and translation structures on the disk, 2013, arXiv:1309.4795.

[11] W. Patrick Hooper, An infinite surface with the lattice property I: Veech groups and coding geodesics, Trans. Amer. Math. Soc. 366 (2014), no. 5, 2625-2649, DOI 10.1090/S0002-99472013-06139-9. MR3165649

[12] W. Patrick Hooper, The invariant measures of some infinite interval exchange maps, Geom. Topol. 19 (2015), no. 4, 1895-2038, DOI 10.2140/gt.2015.19.1895. MR3375521

[13] W. Patrick Hooper, Immersions and translation structures II: Geometric aspects of structures on the pointed disk, 2016, in progress.

[14] W. Patrick Hooper, Immersions and translation structures III: The space of all structures on pointed surfaces, 2016, in progress.

[15] W. Patrick Hooper, Pascal Hubert, and Barak Weiss, Dynamics on the infinite staircase, Discrete Contin. Dyn. Syst. 33 (2013), no. 9, 4341-4347, DOI 10.3934/dcds.2013.33.4341. MR.3038066

[16] W. Patrick Hooper and Richard Evan Schwartz, Billiards in nearly isosceles triangles, J. Mod. Dyn. 3 (2009), no. 2, 159-231, DOI 10.3934/jmd.2009.3.159. MR2504742

[17] Pascal Hubert, Samuel Lelièvre, and Serge Troubetzkoy, The Ehrenfest wind-tree model: periodic directions, recurrence, diffusion, J. Reine Angew. Math. 656 (2011), 223-244, DOI 10.1515/CRELLE.2011.052. MR2818861

[18] Pascal Hubert and Gabriela Schmithüsen, Infinite translation surfaces with infinitely generated Veech groups, J. Mod. Dyn. 4 (2010), no. 4, 715-732, DOI 10.3934/jmd.2010.4.715. MR2753950

[19] Pascal Hubert and Barak Weiss, Ergodicity for infinite periodic translation surfaces, Compos. Math. 149 (2013), no. 8, 1364-1380, DOI 10.1112/S0010437X12000887. MR3103069

[20] K. D. Joshi, Introduction to general topology, A Halsted Press Book, John Wiley \& Sons, Inc., New York, 1983. MR709260

[21] Howard Masur, Interval exchange transformations and measured foliations, Ann. of Math. (2) 115 (1982), no. 1, 169-200, DOI 10.2307/1971341. MR644018

[22] Howard Masur and Serge Tabachnikov, Rational billiards and flat structures, Handbook of dynamical systems, Vol. 1A, North-Holland, Amsterdam, 2002, pp. 1015-1089, DOI 10.1016/S1874-575X(02)80015-7. MR 1928530

[23] C. McMullen, Iteration on Teichmüller space, Invent. Math. 99 (1990), no. 2, 425-454, DOI 10.1007/BF01234427. MR.1031909

[24] Curt McMullen, Amenability, Poincaré series and quasiconformal maps, Invent. Math. 97 (1989), no. 1, 95-127, DOI 10.1007/BF01850656. MR999314

[25] James R. Munkres, Topology, Prentice Hall, Inc., Upper Saddle River, NJ, 2000. Second edition of [ MR0464128]. MR.3728284

[26] David Ralston and Serge Troubetzkoy, Ergodic infinite group extensions of geodesic flows on translation surfaces, J. Mod. Dyn. 6 (2012), no. 4, 477-497, DOI 10.3934/jmd.2012.6.477. MR3008407

[27] David Ralston and Serge Troubetzkoy, Ergodicity of certain cocycles over certain interval exchanges, Discrete Contin. Dyn. Syst. 33 (2013), no. 6, 2523-2529, DOI 10.3934/dcds.2013.33.2523. MR.3007697

[28] Anja Randecker, Wild translation surfaces and infinite genus, Algebr. Geom. Topol. 18 (2018), no. 5, 2661-2699, DOI 10.2140/agt.2018.18.2661. MR3848396 
[29] Martin Schmoll, Veech groups for holonomy-free torus covers, J. Topol. Anal. 3 (2011), no. 4, 521-554, DOI 10.1142/S1793525311000647. MR2887675

[30] J Smillie, The dynamics of billiard flows in rational polygons of dynamical systems, Dynamical systems, ergodic theory and applications 100 (2000), 360.

[31] William P. Thurston, Three-dimensional geometry and topology. Vol. 1, Princeton Mathematical Series, vol. 35, Princeton University Press, Princeton, NJ, 1997. Edited by Silvio Levy. MR 1435975

[32] Rodrigo Treviño, On the ergodicity of flat surfaces of finite area, Geom. Funct. Anal. 24 (2014), no. 1, 360-386, DOI 10.1007/s00039-014-0269-4. MR.3177386

[33] A. N. Zemljakov and A. B. Katok, Topological transitivity of billiards in polygons (Russian), Mat. Zametki 18 (1975), no. 2, 291-300. MR0399423

[34] Anton Zorich, Flat surfaces, Frontiers in number theory, physics, and geometry. I, Springer, Berlin, 2006, pp. 437-583, DOI 10.1007/978-3-540-31347-2_13. MR2261104

Department of Mathematics, The City College of New York, New York, New York, 10031

Email address: whooper@ccny.cuny.edu 